\title{
Optical Wireless Communication for the Internet of Things: Advances, Challenges, and Opportunities
}

\author{
Abdelbaset S. Hamza, Member, IEEE, Tyler Tripp, Student Member, IEEE,
}

\begin{abstract}
The continuous development of the Internet of Things (IoT) calls for innovative solutions and technologies to realize the IoT vision efficiently. One of the rising connectivity technologies that can potentially benefit the IoT deployment is Optical Wireless Communication (OWC) technology. In OWC, light beams from light sources are used to modulate information. The receiver demodulates the received light and processes the signal. The broad unlicensed spectrum of the OWC technology, along with its potential high bit-rate and increased physical link security, motivated researchers to consider OWC for IoT solutions. In this paper, we survey the existing literature related to using OWC technology in the IoT domain. We present the background and preliminaries of the IoT and OWC domains to understand how the OWC fits in the IoT architecture. Then we perform a comprehensive survey of literature related to the use of OWC technology in IoT applications. We highlight and summarize the major papers and experiments in the literature to provide researchers a jump-start to tap into the domain of OWC in IoT using the systemic and detailed survey presented in this paper.
\end{abstract}

Index Terms-Classification, Free Space Optical (FSO), Interent-of-Things (IoT), LED-ID, LED, Optical Camera Communication (OCC), Optical Wireless Communications (OWC), Survey, Wireless Communications.

\section{INTRODUCTION}

$\mathbf{T}$ HE Internet of Things (IoT) is the growing global network of identifiable things (e.g., sensors, actuators, smartwatches, smartphones, and other "smart" devices) connected to the internet. The interconnection of devices creates ecosystems where one device can serve as an access point to others. A hierarchy of devices can collectively operate to inform connected device behaviors and provide insights to users using the continuous data collection by the system. Emerging IoT solutions promise to advance business verticals, impacting different aspects of our daily life, such as health, safety, productivity, and entertainment. As a result, the IoT market is proliferating at a striking rate and the number of things being connected to the Internet is continuously on the rise. Out of the 17B devices connected to the Internet as of 2018, seven billion devices are contributing to the IoT market [1]. By 2025, the number of devices connected to the Internet is expected to be $34.2 \mathrm{~B}$ devices, with $21.5 \mathrm{~B}$ devices being IoT devices (see Figure 1). As the IoT market continues to grow, so does the interest of academia and industry vis-à-vis the IoT domain and solutions.

Wireless communication is usually the technology of choice in IoT access networks to provide the users and things the

A.S. Hamza and T. Tripp are with the Department of Computer Science, Appalachian State University, Boone, NC, 28608 USA (e-mail: hamzaas@appstate.edu; tripptd@appstate.edu).

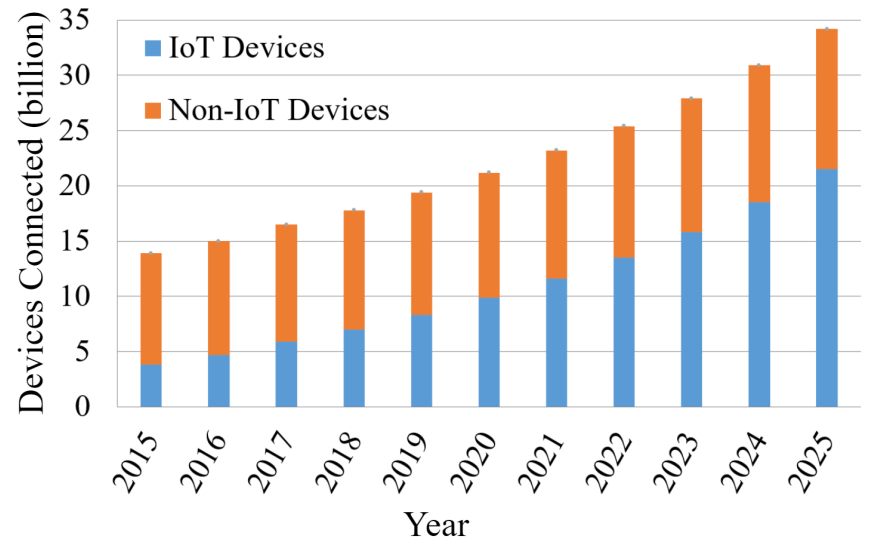

Fig. 1: Total number of active devices connected to the Internet [1]. Non-IoT includes all mobile phones, tablets, PCs, laptops, and fixed line phones. IoT includes all consumer and business-to-business (B2B) devices connected.

full flexibility and mobility needed. According to the visual networking index (VNI) forecast developed by Cisco [2], it is anticipated that the number of public Wi-Fi hotspots and home spots will grow 4-fold to become 549.2 million by 2022 as compared to that of 2017. Moreover, global mobile data traffic is expected to increase seven-fold between 2017 and 2022 to become 77.5 exabytes.

Figure 2 depicts the electromagnetic (EM) spectrum with different frequency ranges (and the corresponding wavelengths) for different wireless technologies. An appropriate carrier frequency is selected based on the application needs. At low carrier frequencies, the long-wavelength allows radio frequency (RF) signals to penetrate walls and windows, diffract over obstacles, and propagate for long-distance due to the low signal attenuation. These advantages of low carrier frequencies, however, come at the cost of the lower bandwidth and thus data rates. On the other hand, increasing the carrier frequency and decreasing the wavelength leads to higher bandwidth and data rates at shorter link range and limited penetration and diffraction.

Most, if not all, IoT wireless access networks currently deployed are utilizing RF communication technologies [3]. The demand for higher data rate communication links is steadily increasing with the development of IoT data-centric applications. To meet these demands, researchers must increase the efficiency of the available congested RF spectrum. A major challenge and the most significant limiting factor of developing higher data-rate RF communication links is 


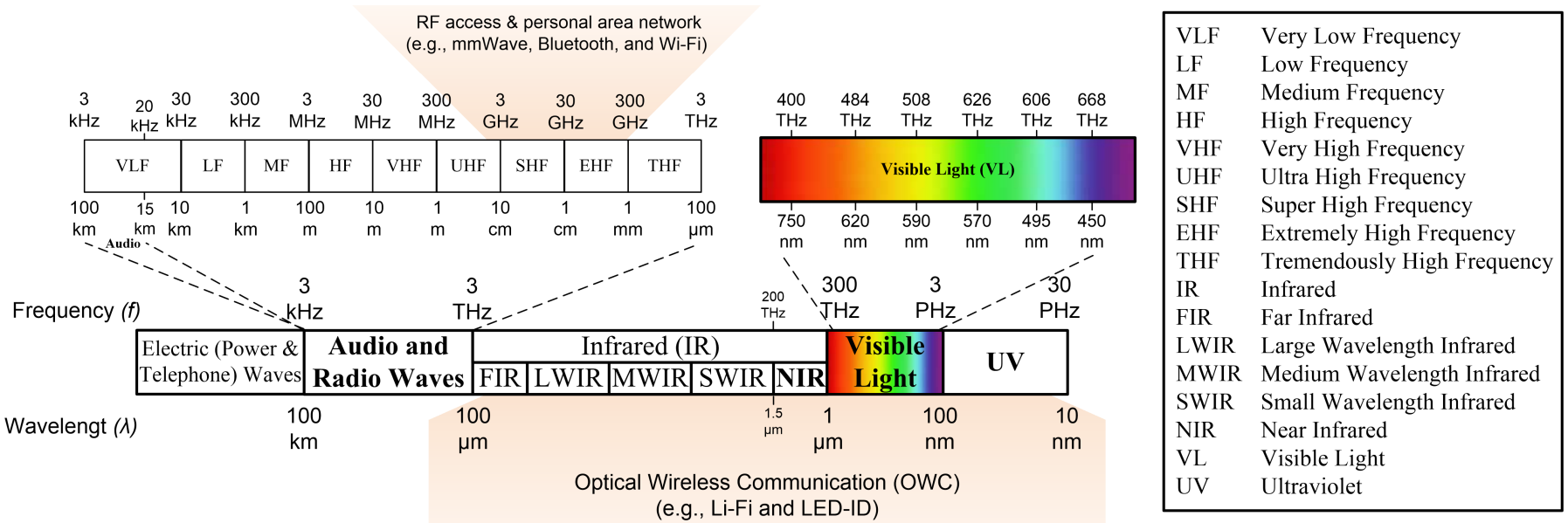

Fig. 2: Abstract overview of a portion of the EM spectrum showing the frequency (and wavelength) ranges for each band. Ranges utilized by both RF and OWC technologies are also indicated. Exact frequency bands allocated for each technology may vary depending on international and national regulatory agencies.

interference [4]. Therefore, the main focus of RF research is on stretching the capability of existing wireless technologies by alleviating interference through coordination.

Nevertheless, RF technologies are being pushed to their limit as we move towards the $5^{\text {th }}$ generation $(5 \mathrm{G})$ cellular wireless system, one of the major enabling technologies for the IoT vision [5]. This is evident by the $2.5 \mathrm{GHz}$ and portions of the $\mathrm{mm}$-Wave band $(30 \mathrm{GHz}-3 \mathrm{THz})$ being cleared and auctioned by the Federal Communication Commission (FCC) for prospective $5 \mathrm{G}$ service providers to allow them to deliver high data rate connections for their customers [6], [7]. The recent advances and development of cost-effective electronics and high-gain steerable antennas operating in the $\mathrm{mm}$-Wave frequency bands, in addition to the higher bandwidth, are the reasons that motivated researchers to explore mm-Wave technology for 5G [8]-[10]. However, at high frequencies, such as in the mm-Wave band, diffraction and reflection of waves barely apply, and the links become limited in range and require higher directivity and Line-of-Sight (LOS). These challenges are currently limiting the wide outdoor deployment of $\mathrm{mm}$ Wave links in 5G networks, and thus are being addressed by the research community [11]. The limitations on the mm-Wave technology outdoor-deployment are considered the motivations for exploring the deployment of indoor short-range high data rate $\mathrm{mm}$-Wave links in other applications such as inter-rack communication in data center networks (DCNs) [12].

Optical Wireless Communication (OWC) is a wireless technology in which a light beam (visible/invisible) is modulated and transmitted to propagate in an unguided medium. It operates in a broad license-free spectrum (see Figure 2) spanning the Infrared (IR), Visible Light (VL), and Ultraviolet (UV) bands. The OWC technology has been gaining increasing attention over the last few decades due to the latest advances in its enabling technologies (light sources and modulation), as well as the fact that it combines the high-bandwidth of fiber optics communications and the flexibility of wireless technologies. As a result, OWC technology continues to find its place in many applications [13] including indoor [12], terrestrial [14], space [15], and underwater [16] applications.

OWC is being considered as a complementary, and sometimes a viable alternative technology to other communication technologies. This encourages the development of hybrid systems in which OWC is joined up with other technologies to improve the overall system performance. For example, OWC can be utilized along with acoustic communication in underwater (UW) wireless sensor networks (WSNs), also referred to as UWSNs [17], [18]. In UWSN, an uncrewed UW vehicle travels between UW sensor nodes to collect data. The vehicle utilizes acoustic signals for long-range localization and accurate ranging. Once the vehicle is aligned with the UW sensor, a high data rate OWC link is established to transfer the data collected by the sensor to the vehicle.

RF communications and OWC are orthogonal mediums, therefore using OWC can help declutter the RF spectrum, reducing interference as more devices are connected. OWC can be combined with RF technology in interference-sensitive applications such as in hospitals and in personal entertainment systems on commercial aircraft that could interfere with navigation and avionics systems [19]. Another important example is the promising role of OWC in the future of access networks in 5G [20]. In a recent study [21], Zhang et. al., propose a hybrid OWC and RF software-defined small-cell networks. Simulation results show improved throughput as a result of the reduced interference in the hybrid system.

The ever-increasing number of IoT devices will require hybrid access network solutions. OWC is a promising technology that is considered as a viable enabling technology for the IoT domain [22]. For example, light sources in OWC transmitters operating in the visible light (VL) band, such as Light Emitting Diodes (LEDs), have the added benefit of serving as illumination sources [23]. This dual-functionality means that the growing abundance of LED sources used worldwide can serve as network access points. On the other end of the OWC link, a high-resolution complementary metal- 
oxide-semiconductor (CMOS) camera, such as the built-in cameras in smartphones and devices can be used to capture the light from the LEDs to establish low data-rate links. The camera-based VLC communication is also referred to as Optical Camera Communication (OCC) which is part of the IEEE 802.15.7-2018 standard developed for Short-Range Optical Wireless Communications [24].

In the context of integrating OWC technologies in IoT networks, some challenges come along with the many advantages OWC technology has to offer. The challenges facing the deployment of OWC in IoT depend on many factors including the specific OWC technology used, application requirements, and the environment in which the system is deployed, i.e., indoor, outdoor, space, or underwater [12]. For example, due to the absence of the penetration and the limited reflection at the high OWC EM bands, OWC requires direct connection via LOS between communication modules to achieve high data rate links. For mobile applications, misalignment becomes a challenge when maintaining a LOS link. On the other hand, the LOS nature of OWC adds a layer of physical security not provided by RF. Other challenges that will be discussed throughout this paper are limited frame rate, synchronization, and ambient light [25]. These challenges have to be addressed to unleash the full potential of OWC technologies in IoT networks.

\section{A. Motivation and Contribution}

It has been noticed that most existing surveys focus on RF technologies when the connectivity technologies in IoT are discussed. On the other hand, technologies such as OWC are rarely discussed and are usually mentioned as a possible technology. This trend is reasonable since RF is widely used, and its enabling technologies are readily available and under continuous research and development. Nevertheless, OWC technology is experiencing rapid development and gaining significant interest in the IoT community. Therefore, we argue that the literature on IoT lags behind OWC development. In this paper, we aim to fill this gap and keep the research community current with the recent advances in OWC technology deployments in the IoT domain.

Being a relatively untapped research space, the scope and boundaries of OWC in IoT are yet to be fully explored. As the application portfolio of OWC technologies in the context of IoT grows, so does the need for a systematic survey. We believe that there is a need for a survey for existing and emerging applications of OWC technologies in the IoT domain. Accordingly, in this paper, we classify and survey all existing work in the area of the OWC in IoT. We aim to give researchers a jump-start to tap into the domain of OWC technology in IoT networks. To improve the readability of the paper, we summarize in Table I all acronyms and abbreviations used in this article.

\section{B. Paper Organization}

The remainder of this paper is organized as follows. In Section II, we provide a brief overview of the IoT with a focus on the related work such as existing classifications and surveys
TABLE I: Acronyms and Abbreviations

\begin{tabular}{|c|c|}
\hline Acronym & Description \\
\hline 6LoWPAN & IPv6 over Low Power Wireless Personal Area Networks \\
\hline AMI & Active Medical Implant \\
\hline AMQP & Advanced Message Queuing Protocol \\
\hline BER & Bit Error Rate \\
\hline BLE & Bluetooth Low Energy \\
\hline $\mathrm{CCD}$ & Charge-coupled device \\
\hline CMOS & Complementary metal-oxide-semiconductor \\
\hline CoAP & Constrained Application Protocol \\
\hline CSK & Color Shift Keying \\
\hline DDS & Data Distribution Service \\
\hline ECG & Electrocardiogram \\
\hline EEG & Electroencephalogram \\
\hline EM & Electromagnetic \\
\hline FCC & Federal Communications Commission \\
\hline FPS & Frames Per Second \\
\hline FSO & Free Space Optics \\
\hline GSM & Global System for Mobile Communications \\
\hline HS-PSK & Hybrid Spatial Phase Shift Keying \\
\hline IIoT & Industrial Internet of Things \\
\hline IoT & Internet of Things \\
\hline IP & Internet Protocol (IPv4: version 4, IPv6: version 6) \\
\hline IR & Infrared \\
\hline LD & Laser Diode \\
\hline LED & Light Emitting Diode \\
\hline LED-ID & Light Emitting Diode Identification \\
\hline LOADng & $\begin{array}{l}\text { Lightweight On-demand Ad-hoc Distance-vector Routing } \\
\text { Protocol - Next Generation }\end{array}$ \\
\hline $\mathrm{LiFi}$ & Light Fidelity \\
\hline LLN & Low-Power Lossy Networks \\
\hline LoRA & Long Range \\
\hline LoRaWAN & Long Range Wide Area Network \\
\hline LOS & Line of Sight \\
\hline LPWAN & Low-Power Wide-Area Network \\
\hline LR-WPANs & Low-Rate Wireless Personal Area Networks \\
\hline LTE-A & Long Term Evolution - Advanced \\
\hline M2M & Machine-to-Machine \\
\hline MQTT & Message Queuing Telemetry Transport \\
\hline MQTT-SN & Message Queuing Telemetry Transport for Sensor Network \\
\hline NFC & Near Field Communication \\
\hline NLOS & Non Line of sight \\
\hline OCC & Optical Camera Communication \\
\hline OOK & On-Off Keying \\
\hline OSC & Optical Scattering Communication \\
\hline OSI & Open Systems Interconnection \\
\hline OWC & Optical Wireless Communications \\
\hline PAN & Personal Area Network \\
\hline PCM & Pulse Code Modulation \\
\hline PD & Photodiode/Photodetector \\
\hline PIN-PD & Positive Intrinsic Negative Photodiode \\
\hline PLC & Powerline Communication \\
\hline RF & Radio Frequency \\
\hline RPL & IPv6 Routing Protocol for Low-Power and Lossy Networks \\
\hline SBC & Single Board Computer \\
\hline TCP & Transmission Control Protocol \\
\hline UDP & User Datagram Protocol \\
\hline UV & Ultraviolet \\
\hline UW & Underwater \\
\hline UWSN & Underwater Wireless Sensor Network \\
\hline V2I & Vehicle to Infrastructure \\
\hline $\mathrm{V} 2 \mathrm{~V}$ & Vehicle to Vehicle \\
\hline $\mathrm{V} 2 \mathrm{X}$ & Vehicle to Everything \\
\hline VL & Visible Light \\
\hline VLC & Visible Light Communication \\
\hline VPPM & Variable Pulse Position Modulation \\
\hline WBFM & Wide Band Frequency Modulation \\
\hline WLAN & Wireless Local Area Network \\
\hline WPAN & Wireless Personal Area Network \\
\hline WSN & Wireless Sensor Network \\
\hline XMPP & Extensible Messaging and Presence Protocol \\
\hline
\end{tabular}




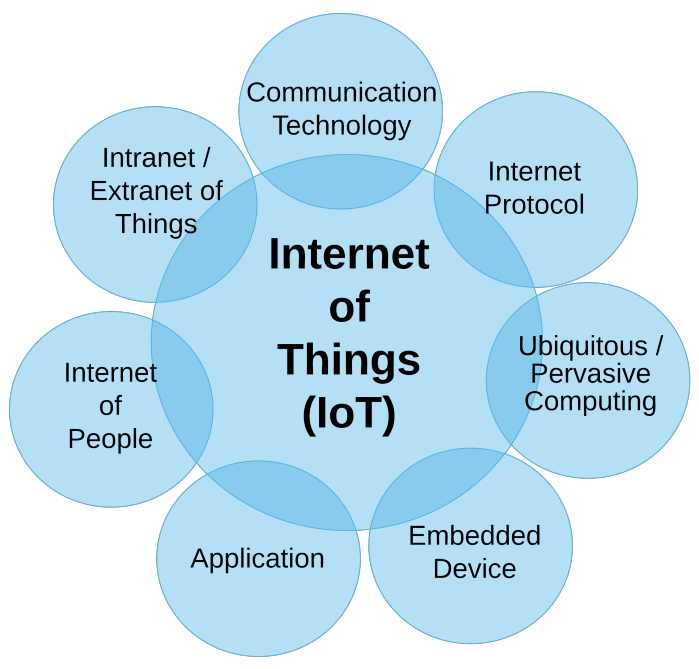

Fig. 3: Scope of the Internet of Things and its relation to other research fields [26]. This figure emphasizes that IoT is not any of these technologies, but rather a overlaps with all of them.

on the IoT. Section III focuses on the preliminaries and basic concepts related to the generic OWC link components, including light sources, photodetectors, and modulation schemes. We dedicate Section IV to classify and review major work in the area of OWC in IoT. Research directions and open problems for OWC in IoT are discussed in Section V. Summary is given in Section VI.

\section{IOT PRELIMINARIES AND BASIC CONCEPTS}

In this section, we cover the preliminaries related to the IoT. This section is necessary to understand how OWC technology can fit into the IoT model. In particular, we discuss the definition of IoT, followed by a brief review of existing taxonomies and surveys.

\section{A. IoT Definition}

Despite the vast body of work in the IoT domain, there is not a definitive agreed-on definition of the term [26]. Different definitions of the IoT exist because each of the defining individuals, organizations, and businesses tend to project their visions and motivations [26]-[28].

In [26], the IEEE IoT Initiative shared a living document with the title "Towards a Definition of the Internet of Things $(I o T)$ " in an attempt to develop an all-inclusive definition of IoT collectively. In this shared document, the collaborators comprehensively survey and review all existing definitions for the term IoT in standards, research projects, national initiatives, white papers, books, and industrial activities. This review did not result in a formulated final definition for the IoT term; instead, the authors recommended a definition that was established by Uckelmann et al. in their edited book entitled "Architecting the Internet of Things" and published in 2011 [28]. For the sake of completeness and to improve the readability of this paper, we include and discuss the definition of IoT presented in [28], which reads as follows:

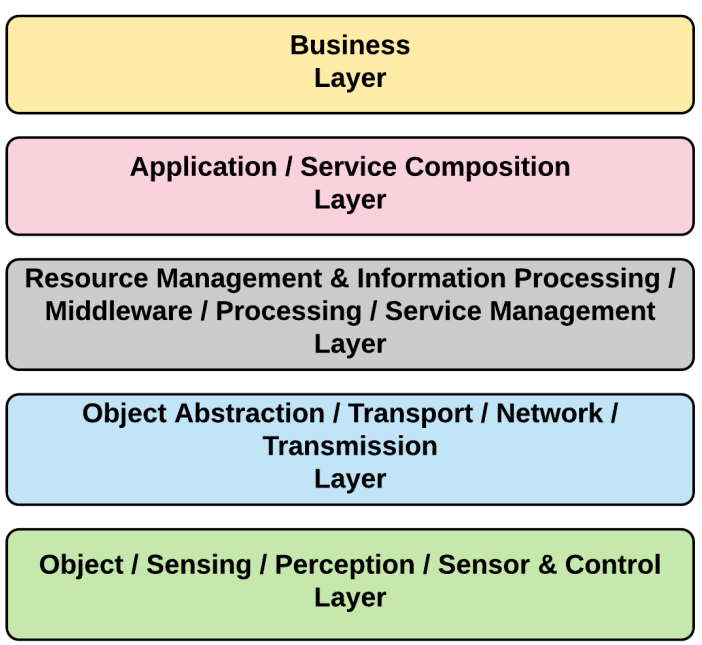

Fig. 4: Five-layer stack-based IoT architecture.

"The future Internet of Things links uniquely identifiable things to their virtual representations in the Internet containing or linking to additional information on their identity, status, location or any other business, social or privately relevant information at a financial or non-financial pay-off that exceeds the efforts of information provisioning and offers information access to non-predefined participants. The provided accurate and appropriate information may be accessed in the right quantity and condition, at the right time and place at the right price. The Internet of Things is not synonymous with ubiquitous/pervasive computing, the Internet Protocol (IP), communication technology, embedded devices, its applications, the Internet of People or the Intranet/Extranet of Things, yet it combines aspects and technologies of all of these approaches."

What makes this definition stand out is that in addition to defining what IoT is, Uckelmann et al. also highlight what IoT is not. Figure 3 [26] depicts the relation between the IoT and other technologies discussed in their definition. Moreover, the definition emphasizes the "rightness" of the different aspects of IoT. The large quantity of data is not the goal; instead, it is the ability to filter and process the data to extract the right amount of data. Data availability does not need to be real-time, but rather at the right time when the data is needed, which implies that it could also be near real-time or even asynchronous. The amount and timing of data must lead to the extraction of the right information appropriate to the user (machine or human) at the right place. The right price for this system is not necessarily the lowest as long as the price is higher than the cost of attaining the information and the possible market price leading to an appropriate revenue margin.

\section{B. IoT Architecture}

Several layered models have been developed to describe the architecture of the IoT as a result of the different visions and 

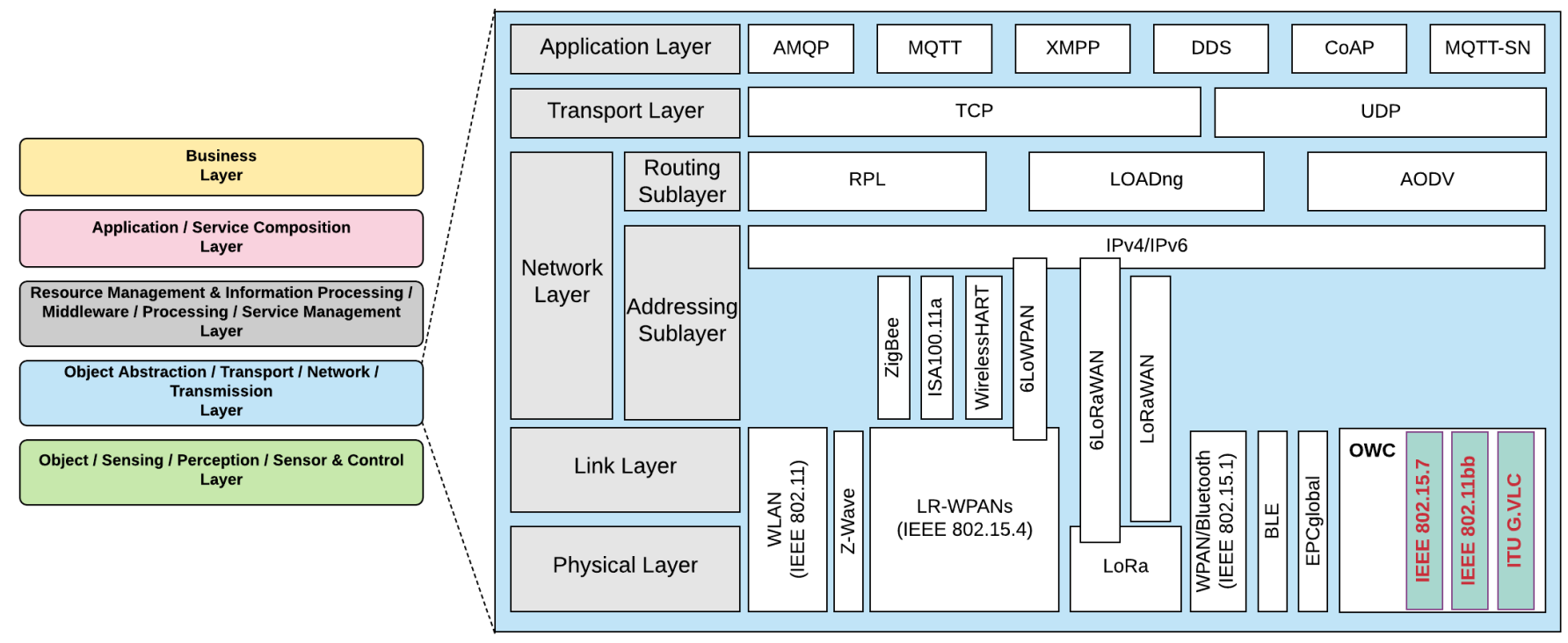

Fig. 5: This figure depicts a partial high-level view of a stack representing the IoT Object Abstraction Layer. Inter-layer protocols' interactions are complex and cannot be demonstrated in a single figure. Therefore, order, size, and alignment of boxes do not signify relationship or importance. Overlapping boxes indicate adaptation layers (i.e., protocols specifically created to adapt two inter-layer protocols.)

objectives of stakeholders [3], [29], [30]. After inspecting the variety of models developed and presented in the literature, we find that the five-layer model (see Figure 4) is the most commonly used model to represent the architecture of the IoT with slight variations from one paper to another. In Figure 4, we list all name variations mentioned in the literature for each layer. In the following, we discuss each layer in detail.

\section{IoT Object Layer}

This is the layer of "things". Since the concept of IoT relies heavily on the sensing and perceiving the surrounding, the layer is often referred to as the "Sensing" or the "Perception" Layer. However, part of the IoT is to take action and actuate [31]. Therefore, we use the name "Object Layer" to refer to this layer [3] since the term object refers to an end-node, i.e., a managed device in the system, whether it is a sensor, actuator, or a hybrid. Dorsemaine et al. present a taxonomy of IoT objects from the attributes prespective [32]. Based on their discussion, an IoT object can be described based on the following attributes; communication, energy, functional attributes, hardware and software resources, and local user interface. In [33], Rozasa et al. present a detailed taxonomy of IoT sensors. They classify IoT sensors into six types, namely; motion, position, environment, mass measurement, and biosensor. An object may or may not be equipped with a connectivity module [34]. Some scenarios in which a sensor performs no communication is when the device is deployed in an environment that requires offline recording and later recovery (e.g., dosimeter). On the other hand, an object equipped with a connectivity module, such as Single Board Computers (SBCs), will transfer the data collected to the higher layer in the architecture through the Object Abstraction Layer.

\section{IoT Object Abstraction Layer}

This layer is responsible for networking the Object and the Middleware layers. The Object Abstraction Layer enables the cross-domain connectivity in which heterogeneous devices are connected to facilitate Machine-to-Machine (M2M) and collaborative interaction [35]. The layer is also responsible for abstracting the output of the object layer and then transmit/transport it up the stack for further processing and vice versa. Depending on the system architecture (Cloud or Fogbased), the task of the Network layer is either to relay the data to a local smart gateway for pre-processing at the edge, or to the cloud where a Middleware performs centralized processing and makes decisions [36].

To relate to conventional networking concepts, we can think of the IoT Object Abstraction Layer as a stack of communication protocols that is partially or fully implementing the Open Systems Interconnection (OSI) model. This layer has its Application, Transport, Network, Link, and Physical layers, which should not be confused with the layers of the IoT architecture. It is worth pointing, however, that large number of technologies and protocols are used in the IoT model. Therefore, one-to-one mapping of all the IoT technologies and protocols to the OSI model is not straight-forward or even possible sometimes. Figure 5 depicts a non-exhaustive overview of the IoT Network Layer with some of the existing protocols, standards, and technologies. The layers in the IoT Network Stack are divided as follows:

1) Application Layer: The various energy, memory, and processing constraints imposed by the IoT objects necessitate the development of more convenient Application Layer protocols as compared to that of the conventional Web application layer protocols. To this end, several IoT-compatible protocols have been developed, namely, Constrained Application 
TABLE II: Comparison of RF protocols for IoT [3], [37], [38].

\begin{tabular}{|c|c|c|c|c|c|c|c|c|c|}
\hline Technology & $\begin{array}{c}\text { PHY } \\
\text { Protocol / Standard } \\
\end{array}$ & $\begin{array}{c}\text { Frequency } \\
\text { Band }\end{array}$ & $\begin{array}{c}\text { Theoretical Data } \\
\text { Rate (bps) }\end{array}$ & $\begin{array}{c}\text { Transmission } \\
\text { Range } \\
\end{array}$ & $\begin{array}{c}\text { Energy } \\
\text { Consumption } \\
\end{array}$ & Cost & $\begin{array}{l}\text { Spreading } \\
\text { Technique } \\
\end{array}$ & $\begin{array}{c}\text { MAC } \\
\text { Access } \\
\end{array}$ & Scalability \\
\hline WSN & IEEE 802.15 .4 & $902-928 \mathrm{MHz}$ & $20-250 \mathrm{~K}$ & $20-100 \mathrm{~m}$ & High & High & NA & NA & NA \\
\hline Wi-Fi & $\begin{array}{l}\text { IEEE } 802.11 \\
\mathrm{a} / \mathrm{c} / \mathrm{b} / \mathrm{d} / \mathrm{g} / \mathrm{n}\end{array}$ & $5-60 \mathrm{GHz}$ & $1 \mathrm{M}-6.75 \mathrm{G}$ & $20-100 \mathrm{~m}$ & High & High & NA & NA & NA \\
\hline WiMAX & IEEE 802.16 & $2-66 \mathrm{GHz}$ & $\begin{array}{c}1 \mathrm{M}-1 \mathrm{G} \text { (fixed) } \\
50-100 \mathrm{M} \text { (Mobile) }\end{array}$ & $<50 \mathrm{Km}$ & Medium & High & NA & NA & NA \\
\hline LPWAN & LoRA & $868 / 900 \mathrm{MHz}$ & $0.3-50 \mathrm{~K}$ & $<30 \mathrm{Km}$ & Very Low & High & NA & NA & NA \\
\hline \multirow{2}{*}{ LR-WPAN } & $\begin{array}{c}\text { Zigbee } \\
\text { IEEE } 802.15 .4\end{array}$ & \multirow[t]{2}{*}{$868 / 915 / 2400 \mathrm{MHz}$} & \multirow[t]{2}{*}{$20 / 40 / 250 \mathrm{~K}$} & $10-20 \mathrm{~m}$ & Low & Low & DSSS & NA & $65 \mathrm{~K}$ nodes \\
\hline & Z-Wave [3] & & & NA & NA & NA & NA & CDMA/CMA & 232 nodes \\
\hline \multirow{2}{*}{ Bluetooth } & IEEE 802.15.1 & \multirow{2}{*}{$2.4 \mathrm{GHz}$} & $1-24 \mathrm{M}$ & \multirow{2}{*}{$8-10 \mathrm{~m}$} & Medium & \multirow{2}{*}{ Low } & FHSS & TDMA & 5917 slaves \\
\hline & BLE & & $1024 \mathrm{~K}$ & & Very Low & & NA & NA & NA \\
\hline \multirow{2}{*}{ RFID } & EPCglobal & $860-960 \mathrm{MHz}$ & $5640 \mathrm{~K}$ & NA & NA & NA & DS-CDMA & ALOHA & NA \\
\hline & ISO/IEC 15,693 & $860-960 \mathrm{MHz} 2.4 \mathrm{GHz}$ & $106 \mathrm{k}-424 \mathrm{~K}$ & $<100 \mathrm{~m}$ & Medium & Medium & NA & NA & NA \\
\hline $\begin{array}{c}\text { Mobile } \\
\text { Communication }\end{array}$ & $\begin{array}{c}\text { LTE-A } \\
\text { 2G-GSM,CDMA } \\
\text { 3G-UMTS,CDMA2000 } \\
\text { 4G-LTE } \\
\text { 5G }\end{array}$ & $\begin{array}{c}865 \mathrm{MHz} \\
2.4 \mathrm{GHz} \\
3.5 \mathrm{GHz} \\
26 \mathrm{GHz}\end{array}$ & $\begin{array}{c}\text { 2G: } 50-100 \mathrm{~K} \\
\text { 3G: } 200 \mathrm{~K} \\
\text { 4G: } .1-1 \mathrm{G} \\
\text { 5G: } .25-10 \mathrm{G}\end{array}$ & $\begin{array}{c}\text { Entire } \\
\text { Cellular } \\
\text { Area }\end{array}$ & Medium & Medium & Multiple CC & OFMDA & NA \\
\hline
\end{tabular}

Protocol (CoAP) [39], Message Queue Telemetry Transport (MQTT) [40], Extensible Messaging and Presence Protocol (XMPP) [41], Advanced Message Queuing Protocol (AMQP) [42], Data Distribution Service (DDS) [43], and Web Socket [44], [45]. Al-Fuqaha et al. discuss different IoT Network Application Layer protocols in detail [3]. In [45], Karagiannis et al. present a comparison of different Application Layer protocols with respect to different metrics: Transport protocol, Quality of Service options, Architecture, and Security.

2) Transport Layer: This layer provides host-to-host communication services for the applications used. These services include connection-oriented communication, reliability, flow control, and multiplexing. The two most popular Transport Layer protocols are adopted from the Internet protocol suite, namely, Transmission Control Protocol (TCP) and User Datagram Protocol (UDP). TCP is a connection-oriented protocol that provides reliable transmission, whereas UDP is a connection-less protocol with no guarantees of data delivery. For example, MQTT, XMPP, and AMQP Application Layer protocols utilize TCP. On the other hand, the CoAP protocol is a UDP protocol. A different specification for MQTT protocol for Sensor Networks (MQTT-SN) was developed to map the MQTT to operate with UDP. The DDS protocol is designed to operate with both TCP and UDP [46].

3) Network Layer: This layer is responsible for addressing objects and routing data between different entities in the network. It was envisioned that the IPv6 would help solve the challenges related to the addressing of a large number of objects in the IoT model. However, as the constraints of IoT objects continue to propagate through the stack of the IoT Object Abstraction Layer, utilizing IPv6 could prove cumbersome for some of the IoT objects. To address the limitation of using IPv6 with low-power devices, a modified version of the IPv6 in which overheads are removed has been developed as part of the Low-Rate Wireless Personal Area Networks (LR-WPANs). This modification has led to the development of IPv6 for Low power Wireless Personal Area Networks (6LoWPAN) protocol [47]. Other LR-WPANs protocols include Zigbee [48], ISA100.11a [49], and WirelessHART [50]. Long Range Wide Area Network (LoRaWAN) protocols are being developed for IoT. The protocols are operating on top of the Long Range (LoRa) Physical Layer protocol. An adaptation of LoRaWAN has been developed to allow for IPv6 transmission, referred to as 6LoRaWAN [51].

Routing protocols operate on top of the addressing protocols to forward data from one node to another. Depending on the network segment responsible for forwarding the data, different routing protocols will be used. For example, the ZigBee network utilizes the Ad hoc On-Demand Distance Vector (AODV) Routing protocol [52].

Routing protocols developed for IoT Low-Power Lossy Networks (LLNs) can be classified as proactive or reactive. In proactive routing protocols, gateways construct routes between nodes in the network even without data routing requests [53], making these routing protocols suitable for applications with periodical data collection. A famous example of proactive routing protocols used for is the IPv6 Routing Protocol for Low-Power and Lossy Networks (RPL) [54]. On the other hand, a reactive routing protocol establishes on-demand routes when a node has data to transmit. Therefore, a reactive routing protocol is most suitable for applications with infrequent data traffic. An example of a reactive routing algorithm for LLNs is the Lightweight On-demand Ad hoc Distance-vector Routing ProtocolNext Generation (LOADng) [55] and LOADng-IoT [53].

4) Link Layer: This layer is responsible for taking the data formatted by the Network Layer and prepare it for the singlehop node-to-node transmission through the Physical Layer. Protocols at this layer are directly related to the protocols and technologies used in the Physical Layer. Therefore, for most of the conventional wired and wireless technologies utilized by the IoT network, the corresponding Link Layer protocols, including logical link control (LLC) and media access control (MAC) sublayers, are pre-defined. New technologies developed are being defined and usually protocols for Link and Physical Layers are jointly defined.

5) Physical Layer: The Physical Layer (PHY) is responsible for the bit-level transmission of data over the physical medium. The medium used in this layer can be wired or wireless. Wired medium provides high capacity; however, it 


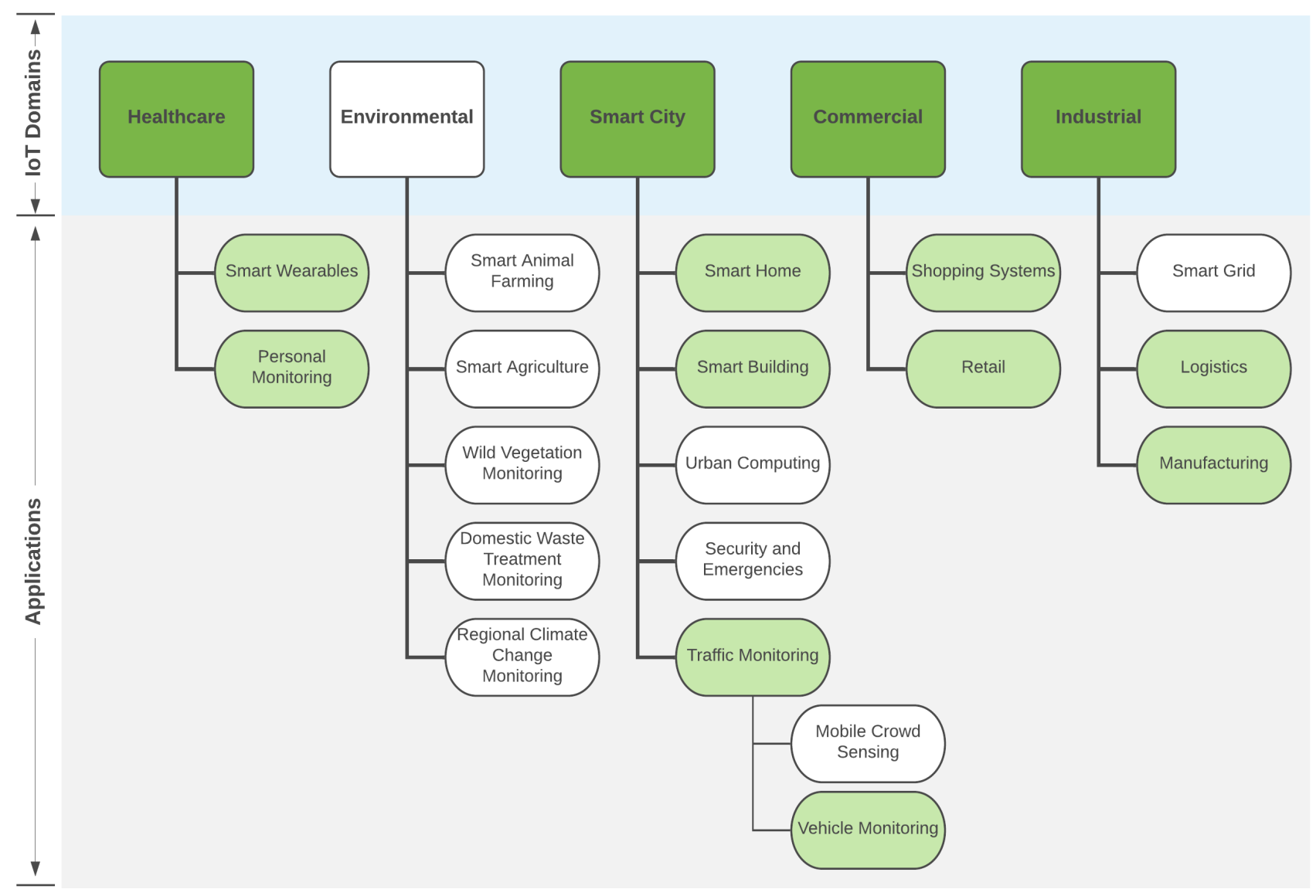

Fig. 6: A taxonomy of IoT domains and applications adopted from [56] with some modifications. Highlighted blocks indicate domains and applications for which at least one paper that discusses an OWC link is found and surveyed.

has limited flexibility and can be costly. On the other hand, wireless communication provides flexibility and mobility.

There is a wide range of RF Wireless technologies, and thus, considerations for range, reliability, bandwidth, security, cost, energy consumption, and environmental factors influencing which protocol to use [3], [37], [38], [56]. Table II summarizes different wireless protocols and technologies used in the Physical Layer of the IoT. The IEEE 802.15.4 and IEEE 802.11 standards are the basis for several Object Abstraction Layer protocols that utilize IP routing. IEEE 802.15.4 focuses on the Personal Area Networks (PANs) with short-range, lower power, and lower data rates as compared to the IEEE 802.11. WiMAX (IEEE 802.16) is a standard similar to Wi-Fi but has access to lower frequencies that are better at penetrating obstacles. The Cellular network is a widely used network with worldwide coverage and an ability to maintain the connection in highly mobile applications. LoRa and Z-Wave are low data rate, low power consumption RF protocols that operate over a large coverage area using sub $\mathrm{GHz}$ frequencies commonly used in Wireless Sensor Networks (WSNs). Bluetooth is used to create PANs while utilizing management features not present in other protocols using the same frequency band. Bluetooth Low Energy (BLE) is a lower energy implementation that operates around the same range but at a lower data rate. RFID is suitable for identifying objects since it facilitates a low-power one-way connection that sends only a single message called a tag or ID to a nearby receiving device.

OWC technologies, which are the focus of this survey, span the three lower layers (Physical, Link, and Network Layers) in the IoT Object Abstraction Layer with varying degrees depending on the specific OWC technology. Therefore, in the rest of this paper, our discussion will focus on the Object Abstraction Layer. The most significant OWC standard that is related to the IoT is the IEEE 802.15.7, which defines the protocols for the Physical and Link Layers. The standard will be discussed in detail in Section III as we present the preliminaries and basic concepts for the OWC domain.

\section{IoT Middleware}

The scale of diversity at the lower layers of the IoT architecture and thus the accompanying challenges become more apparent as we move up the stack. One such difficulty is the fact that no common standard can be developed and used with the variety of devices deployed in different IoT domains [57]. To develop a meaningful business model, various applications 
from different domains must utilize the data sent from the perception layer. To this end, data must be abstracted and adapted to be ready for different applications [29]. The Middleware IoT layer is the layer responsible for performing this abstraction to interface the diverse and heterogeneous components and services at lower layers and the IoT Application layer [3], [29], [57]. The Middleware layer performs its tasks by storing in databases, analyzing, and processing the massive scale of data collected by the sensors and relayed by the object abstraction layer. Data collected is then used to develop services through mashup tools. The mashup tools provide the users at the IoT Application layer with application programming interfacing (API) to utilize the services. The degree of sophistication in the mashup can range from putting data together in a manually prewired fashion to using semantics and ontology leading to Semantic Mashup [58].

\section{IoT Application Layer}

This layer is the layer at which services from IoT's infrastructure along with the industry needs converge to realize the intellectualized industry; that is, the conventional industry with the added value of processed data from the IoT Middleware [29]. Applications can be in a wide range of sectors and domains such as healthcare, smart city, commercial, environmental, telecommunications, and industrial, among others. In the following, we briefly summarize the major IoT domains discussed in this paper:

- Healthcare: IoT connected devices used by healthcare providers to monitor and manage patient health with rapid, accurate, low-cost solutions are increasing. The Healthcare domain comprises any application that is related to maintaining or monitoring patient health.

- Smart City: Applications involve both public and private sector applications that do not pertain to sales or production. Managing resources for a building, transportation, or public area constitute a general goal for Smart City applications.

- Commercial: Applications aim to attain economic goals. Usually involves improving user experience in facilities and areas such as shopping centers and museums.

- Environmental: Applications pertain to agricultural and natural spaces and resources such as water, energy, and mining.

- Industrial: Includes applications related to manufacturing, production, and efficient distribution of resources, like running factory equipment or managing a supply chain for a warehouse.

Figure 6 depicts a summary of IoT domains and applications. It is worth pointing out that the domains and applications taxonomy presented in Figure 6 is non-exhaustive and is not based on standard classification. Therefore, there may be overlaps between different applications. It is also possible that one application can be thought of from different perspectives such as provider and consumer. As a result, the same application can be listed under two different domains.

\section{IoT Business Layer}

At this layer, the services provided by a business or a vertical converge with the needs of the business customers (individuals or other businesses). Among others, the responsibility of the IoT Business layer is to develop a profitable business model [29], [30] taking into consideration the cost of collecting, relaying, and processing data in the underneath IoT layers. The cost of developing market analysis reports, application and business services, marketing, and managing and securing users' data are also considered at this layer.

\section{Existing IoT Taxonomies and Surveys}

The lack of a unified IoT definition has led to the existence of several IoT taxonomies and classification schemes in the literature, none of which is inclusive nor agreed-upon. Most existing taxonomies either list different classes at each of the layers in the IoT stack [34], [36], [59], [63] or focus on one or a subset [32], [33], [56], [57] of the layers in the IoT architecture. In this paper, we follow the former approach of using the full stack of IoT layers to describe a surveyed system from the literature in Section IV.

In [33], Rozsa et al. present a taxonomy of sensors at the Object Layer based on the domains at the Application Layer. In [32], Dorsemaine et al. present a more generic taxonomy that can fully characterize any connected object in the IoT Object layer. An object is characterized by its communication, functional attributes, hardware and software resources, local user interface, and energy. A taxonomy of networking technologies used in the Object Abstraction layer is presented by Sethi and Sarangi in [36]. In this paper, the authors classify networking into Wireless Sensor Networks (WSNs), Near Field Communication (NFC), RFID and WSN integration, Internet Protocols for Smart Objects, and LowPower Technologies. Multiple surveys focused on the Middleware layer [57], [58], [64]. General Application layer domains are classified in [56].

Similarly, there is a significant number of survey papers on the IoT. Some of the surveys in the literature review IoT architectures [37], security [58], protocols [3], middlewares [57], [64], general applications [56], or specific applications such as, smart cities [62] and IoT in healthcare domain [38]. Table III tabulates major and most related IoT taxonomies and surveys in the literature.

Al-Fuqaha et al. conducted a survey on IoT protocols, with a limited discussion about specific use-cases. The Object Abstraction layer protocols examined for use in IoT networks were all lightweight RF link layer protocols [3]. IEEE 802.15.4, BLE, EPCglobal, Cellular, and Z-Wave were examined across five characteristics to compare feasibility in IoT systems. From these specifications, the authors determined that the efficient communication and scalability of these protocols were the most attractive link layer protocols to use in IoT and M2M environments [3].

In [37], Ray surveys 130 research papers to identify characteristics of IoT architecture. This included use-cases, protocol stacks, and grouping these into application domains to discover trends. With regards to RF in IoT, the author assessed that 
TABLE III: Chronological Summary of Major and Related IoT Taxonomies and Surveys in the Literature.

\begin{tabular}{|c|c|c|c|c|c|c|}
\hline Reference & Year & Type & IoT Layer & Focus & $\mathbf{R F}$ & OWC \\
\hline Xu et al. [60] & 2014 & Survey & Multi & $\begin{array}{c}\text { Survey of } \\
\text { technologies } \\
\text { and applications }\end{array}$ & $\checkmark$ & $x$ \\
\hline Al-Fuqaha et al. [3] & 2015 & Survey & Multi & Protocols & $\checkmark$ & $x$ \\
\hline Kadam and Dhage [61] & 2016 & $\begin{array}{l}\text { Literature } \\
\text { Review }\end{array}$ & NA & $\begin{array}{c}\text { Modulation } \\
\text { techniques }\end{array}$ & $x$ & $\checkmark$ \\
\hline Rozsa et al. [33] & 2016 & Taxonomy & $\begin{array}{l}\text { Application } \\
\& \text { Object }\end{array}$ & $\begin{array}{l}\text { Classification of } \\
\text { sensors based } \\
\text { on applications }\end{array}$ & $x$ & $x$ \\
\hline Arasteh et al. [62] & 2016 & Survey & Application & Smart Cities & $x$ & $x$ \\
\hline Yaqoob et al. [63] & 2017 & Taxonomy & Multi & $\begin{array}{l}\text { Multi-layer } \\
\text { classification }\end{array}$ & $\checkmark$ & $x$ \\
\hline Sethi and Sarangi [36] & 2017 & $\begin{array}{l}\text { Taxonomy } \\
\text { \& Survey }\end{array}$ & Multi & $\begin{array}{l}\text { Multi-layer } \\
\text { survey }\end{array}$ & $\checkmark$ & $x$ \\
\hline Ngu et al. [64] & 2017 & Survey & Middleware & $\begin{array}{l}\text { Survey of } \\
\text { Middlewares }\end{array}$ & $\checkmark$ & $x$ \\
\hline Ahmadi et al. [38] & 2018 & Survey & Application & Healthcare & $\checkmark$ & $x$ \\
\hline Teli et al. [22] & 2018 & $\begin{array}{l}\text { Literature } \\
\text { Review }\end{array}$ & NA & $\begin{array}{c}\text { General } \\
\text { Applications } \\
\text { and Challenges }\end{array}$ & $x$ & $\checkmark$ \\
\hline Hou et al. [65] & 2019 & Survey & Multi & Security & $x$ & $x$ \\
\hline Turan et al. [66] & 2019 & Survey & Application & $\begin{array}{l}\text { VLC technology } \\
\& \text { future } \\
\text { directions }\end{array}$ & $x$ & $\checkmark$ \\
\hline Chowdhury et al. [67] & 2020 & $\begin{array}{c}\text { Classification } \\
\text { and Survey }\end{array}$ & $\begin{array}{l}\text { Application } \\
\text { and Object } \\
\text { Abstraction }\end{array}$ & $\begin{array}{c}\text { Review of } \\
\text { Connectivity } \\
\text { Technologies }\end{array}$ & $\checkmark$ & $\checkmark$ \\
\hline
\end{tabular}

the most common communication protocols within the surveyed use-cases were WiFi, WiMAX, IEEE 802.15.4, Cellular, Bluetooth, RFID, and LoRa. The surveyed IoT architectures were classified across domains including RFID, WSNs, Supply Chain Management, Healthcare, Smart society, Cloud services, Social computing, and Security.

In [56], Asghari et al. selected 72 research studies in IoT applications then focused on categorizing the domain of IoT applications and identifying a standard taxonomy amongst them. Out of that sample, the authors determined the evaluation environments for the IoT application and found that $24 \%$ were implementations, $58 \%$ contained data from simulations, and $14 \%$ had neither. However, there was a lack of focus on protocol stacks, with only 11 Object Abstraction protocols identified and five Application Layer protocols listed. Out of the Object Abstraction protocols, all were RF standards and included 3G, BLE, ZigBee, GSM, WiFi, RFID, 802.15.4, and Narrowband RF.

Ahmadi et al. surveyed 60 research papers that focused on IoT applications within the Healthcare domain to find the effects and challenges of IoT in healthcare [38]. The paper included 3 Application layer protocols, and 8 Object Abstraction layer protocols in the overview and detailed which protocols used within all surveyed Healthcare applications.

Despite being incomprehensive, Table III shows that the majority of existing IoT survey papers addressing the wireless 
communication in the IoT Object Abstraction layer focus on the RF technologies. The focus on RF technologies is justifiable since RF technologies are the most widely used wireless technologies and have been under continuous development for many decades. On the other hand, very few papers have surveyed the deployment of optical communication technologies in general, and the OWC technology in particular, in the IoT domain. Even within the optical communication research community, papers discuss the deployment of fiber optics in the backbone of the IoT infrastructure [68] with no or limited discussion of the OWC technology. On the other hand, the papers that address the OWC technology in IoT are limited to the enabling technologies of the OWC itself or high-level discussions of the potential of the OWC in IoT.

In [61], Kadam and Dhage present a literature review of VLC and hybrid VLC/RF setups in general used to advance the VLC domain. Two of the surveyed papers are related to IoT applications. Surveyed papers are discussed with a focus on identifying modulation techniques that are most suited for indoor applications, including IoT. However, papers that used OWC in IoT specific applications were not reviewed.

In [22], Teli et al. present a high-level discussion of the advantages and the potential role of the VLC technology in different applications that can fall under the IoT domain, referring to it as the Optical Internet of Things (OIoT). The authors review five papers discussing enabling technologies for VLC in IoT, including protocols, localization, and modulation. The paper, however, does not get into the details of specific applications and implementations.

In [66], Turan et al., provide a summary of the role of VLC in the Industrial Internet of Things (IIoT). Similar to the work in [22], the majority of the book chapter focuses on the VLC domain and its enabling technologies. The paper then highlights the potential of the OWC in future IoT applications. A total of three papers related to the OWC in IoT were reviewed by the authors.

In [67], Chowdhury et al. present a visionary discussion of the anticipated role of the OWC technologies in the $5 \mathrm{G} / 6 \mathrm{G}$ and IoT solutions. The paper reviews the $5 \mathrm{G}, 6 \mathrm{G}$, and IoT systems and requirements followed by a short review of existing OWCBased 5G and IoT Systems, out of which four reviewed papers are related to the IoT.

In a more recent paper [69], Ding et al. review connectivity technologies and applications in IoT. This paper is the first paper to discuss OWC as an integral part of the connectivity in the Object Abstraction Layer in the IoT. The paper, however, had a minimal review of OWC deployments in the IoT domain in which the work by Teli et al. [22] is referenced.

There is an abundance of publications in general, and surveys in particular, discussing the IoT. Limited survey papers focus on OWC technology in the context of IoT. None of the OWC-centric survey papers discuss OWC as an enabler of a complete IoT solution with a clear understanding of the object, object abstraction, middleware, and application layers. Instead, the majority of the publications focus on the VLC as an OWC technology, high-level visionary discussions of VLC in IoT, and VLC enabling technologies such as modulation and localization.

\section{OWC PRELIMINARIES AND BASIC CONCEPTS}

Based on the IoT five-layer model discussed in Section II, we can see that the OWC fits in the Object Abstraction layer with other RF technologies and standards, such as RFID, 5G, WiFi, Bluetooth Low Energy, etc. Unlike RF, OWC has fewer standards with the most notable standard being the IEEE 802.15.7-2018 [13], [70]. Therefore, in the case of OWC application in IoT, we cannot only mention the standard as is the case for RF. Instead, we believe that a detailed and more specific classification must be used to distinguish OWC implementations in different IoT systems.

In this section, we present a brief background related to OWC technology. This background is necessary to understand the technical contents of the papers surveyed in Section IV.

\section{A. OWC Frequency Bands and Link Configurations}

OWC is the use of a modulated fast switching light emitter such as a Light Emitting Diode (LED) or Laser Diode (LD) to send data that can be received by image sensors (e.g., camera) or photodetectors [13]. The three frequency bands of Infrared (IR) $3 \mathrm{THz}-300 \mathrm{THz}$, Visible Light (VL) $380 \mathrm{THz}-790 \mathrm{THz}$, and Ultraviolet (UV) $790-3000 \mathrm{THz}$ are commonly used to break up the large OWC bandwidth into frequencies with similar characteristics. Similar to fibre-optic systems, Near IR (NIR) band is used in OWC applications in almost all environments indoor [12], underwater [71], terrestrial, and space [72]. UV is also invisible and can be physiologically damaging because of its high energy [13], therefore, UV is proposed to be used in terrestrial and underwater OWC systems using back-scattering links which is referred to as optical scattering communication (OSC) [73]. VL is the only visible band to the naked eye and is utilized widely to illuminate living quarters, work spaces, and businesses. It is proposed to utilize the growing LED-based illuminating infrastructure to perform communication along the primary illumination functionality leading to the VL Communication (VLC) that is widely used in indoor [74], terrestrial [75], and underwater [16].

An OWC link can be classified based on the following five criteria [13]:

- Environment $(\varepsilon)$ : OWC technology can be used in four different environments, namely: Indoor (I), Terrestrial $(T)$, Space $(S)$ and Underwater $(U W)$.

- Coverage Type $(\kappa)$ : An OWC link can be either pointto-point, i.e., Point Coverage (PC) or point-to-multipoint, i.e., Cellular Coverage (CC) link.

- LOS Availability $(\alpha)$ : An OWC link can be achieved using $L O S$ or non-LOS $(N L O S)$ link configuration. In case of the NLOS OWC link, the the light can either reflect of off a specular surface (e.g., mirrors, beam splitters) or a relayed using an active repeater that receives a signal from the transmitter and re-transmits the signal to the intended receiver. Due to the limited reflectivity of the high-frequency OWC waves, the NLOS model adds challenges to the modeling and practicality of the OWC applications. 


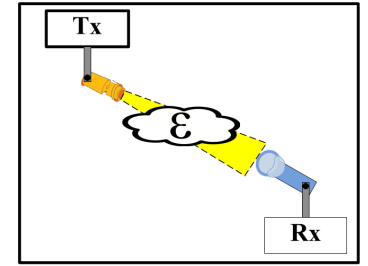

$\varepsilon / P C / L O S / F / X$

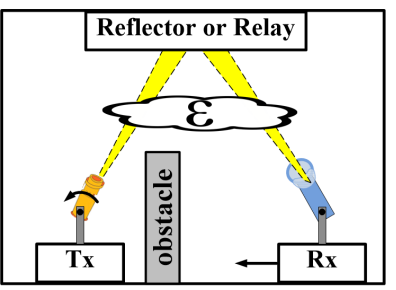

$\varepsilon / P C / N L O S / M / x$

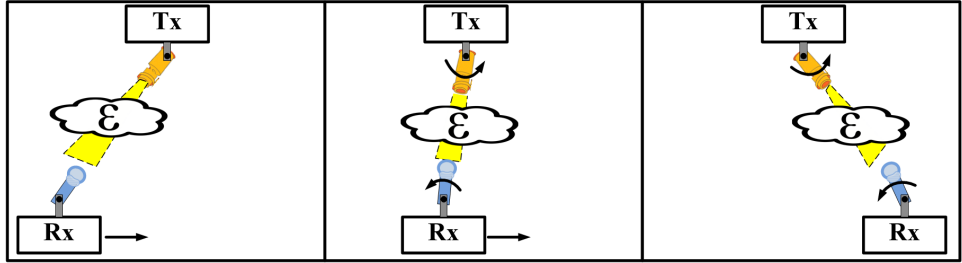

E/PC/LOS/M/X

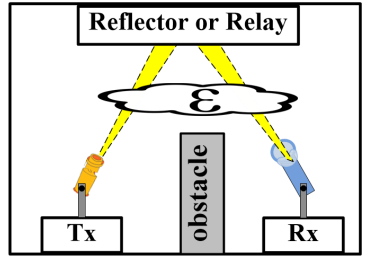

$\varepsilon / P C / N L O S / F / x$

Fig. 7: Different OWC link configurations based on the classification in [13]. The cloud symbol represents the environment $(\epsilon)$ in which the link is implemented. The $\epsilon$ can represent Indoor (I), Terrestrial (T), Space (S), Underwater (UW), or anay combination of these environments. We use the notation $T x$ and $R x$ to refer to a transmitter and a receiver, respectively. Arrows represent mobility of the transmitter and/or receiver in any direction.

- Mobility $(\mu)$ : Depending on the mobility of the transmitter and receiver, an OWC link can be either a fixed $(F)$ or mobile $(M)$ link.

- Link Distance $(\delta)$ : The range of the OWC link can be Ultra-short range $[\leq 5 \mathrm{~cm}$ e.g., chip-to-chip communications], Short range $[\leq 50 \mathrm{~m}$ e.g., underwater communications], Medium range $[\leq 500 \mathrm{~m}$ e.g., indoor wireless local area networks (WLANs)], Long range [ $\leq 500 \mathrm{~km}$ e.g., terrestrial connections], and Ultra-long range [ $\geq 500 \mathrm{~km}$ e.g., deep space links].

Using these five criteria, an OWC link configuration can be expressed using the tuple $(\varepsilon / \kappa / \alpha / \mu / \delta)$, such that:

$$
\begin{aligned}
& \varepsilon \in \mathcal{P}(\{I, T, S, U W\}) \backslash\{\emptyset\} . \\
& \kappa \in\{P C, C C\} \\
& \alpha \in\{L O S, N L O S\} \\
& \mu \in\{F, M\} \\
& \delta \in\{\text { UShort, Short, Medium, Long, ULong }\}
\end{aligned}
$$

where $\mathcal{P}($.$) is the power set. For example, an indoor, point-$ to-point link that is established between two fixed terminals that are few meters apart and utilizes a LOS model can be described as $I / P C / L O S / F / M e d i u m$. Figure 7 depicts different link configurations that result from the classification adopted in the generic environment $\varepsilon$.

The choice of the transmitter, receiver, and modulation technique depend heavily on the application requirements and constraints. In the following, we briefly describe different types of transmitters, receivers, and modulation schemes to familiarize the reader with the OWC technology.

\section{B. Transmitters}

An OWC link can utilize a single or an array of light sources at the transmitter. A light source can be either a Laser Diode
(LD) or a Light Emitting Diode (LED). In the following, we briefly discuss both light sources.

$L D$ : an LD emits high-bandwidth coherent and focused beams with low divergence. Therefore, LDs are usually used for high data rate specialized indoor and long-distance outdoor applications. The high-power focused beams from LDs, however, can be harmful to the eyes, and therefore regulations for operating power are in place. A possible approach to comply with the restrictions when operating using LD is to deploy a beam diffuser to reduce the intensity and spread the beam.

LED: Compared to LDs, LEDs are usually cheaper to produce and more stable. LEDs are also less hazardous to humans than LDs since they are extended sources with larger emitting areas and beams. The diffused LED beam, however, leads to lower signal strength, and thus relatively shorter achievable distance and data rate [76]. Recent research efforts aim to increase the data rate of LEDs recent studies show that Organic LEDs (OLEDs) can help achieve high data rates up to 1.13 Gbps [77]. Another study by Tsonev et al. demonstrates a 3 Gbps FSO link utilizing a single $50-\mu m$ gallium nitride LED and Orthogonal frequency division multiplexing (OFDM) modulation scheme [78].

In the context of IoT, an OWC link will more likely utilize an LED over an LD [79]. This is because LEDs are cheaper and more reliable as compared to LDs. Moreover, LDs emit narrow beams leading to lower coverage and stringent alignment requirements. On the other hand, LEDs are extended sources with large-area emitters leading to broader coverage areas and safe operations at relatively high power. Using an LED light source, a transmitter can send data that is processed $L E D$, or unprocessed LED Identification (LED-ID). With that said, there is not a technical reason that hinders the use of the LD in the IoT domain. At the other end of the IoT OWC link, the data transmitted can be received using 
photodiodes (PDs) or with cameras which is called Optical Camera Communication (OCC).

\section{Receivers}

Receivers in OWC systems can utilize photodiodes (PDs), also referred to as photodetectors, or cameras to detect transmissions. In the following, we briefly discuss both types of OWC receivers.

Photodiodes (PDs): The two most common PD receivers are Positive-Intrinsic-Negative (PIN) diodes and Avalanche photodiodes (APDs). All PDs absorb photons and generate a current in proportion to the absorption with different degrees. Because of the fast switching of the generated current, PDs are desirable for high bit rate transmissions [95].

PINs are low-cost PDs that can operate at low bias and are tolerant to wide temperature fluctuations [96], [97]. Therefore, PIN PDs are preferred in low-cost and low data rate OWC links. APDs are PIN PDs operating at very high reverse bias. As a result, APDs experience high internal electrical gain and Signal-to-Noise Ratio (SNR) at the receiver [96], [98]. The additional gain generated in the APDs makes them excellent candidates for applications with limited ambient light noise and in high data rate and high-performance OWC links. APDs, however, are more expensive, and their gain is temperaturedependent. In [97], Ghassemlooy et al. analyze different noise sources impacting PINs and APDs.

Researchers are exploring technologies such as graphene, two-dimensional materials, and (nano)materials, such as plasmonic nanoparticles, semiconductors, quantum dots to develop faster PDs capable of meeting the demands for higher data rate links [99]-[101].

Recent research, also, explores replacing or integrating the PDs with solar panels [102]. In this scenario, solar panels will simultaneously serve as energy harvester and PD, leading to a prolonged operation lifetime. The extended operation time is a useful feature for IoT applications. To distinguish regular PDs and those equipped with solar panels, we refer to the latter as Photodiode-Solar Panel (PD-S) receivers.

Image Sensors: A typical example of image sensors is the complementary metal-oxide-semiconductor (CMOS) camera that is built-in most of the consumer-grade electronic devices. Such cameras can be used as receivers in OWC links [13]. If a camera is used, the OWC link is referred to as an Image Sensor Communication (ISC), or more commonly as an Optical Camera Communication (OCC) link. OCC is more practical for most consumer-specific applications because it allows consumer-grade devices, that are not specialized in OWC, to receive data through a CMOS camera with rolling shutter. OCC technology offers several advantages such as the larger field of view (FOV), spatial separation of light, and wavelength separation [103]. We refer readers interested in OCC technology to the recent work by Saeed et al. [104] in which they present a comprehensive survey of the technology. Other related work can be found in [105]-[108].

A camera shutter can be either a rolling shutter (RS) or a global shutter (GS). The difference between the two shutter types is how the camera captures an image. Rolling shutter develops an image by capturing one row of pixels at a time, working across the frame, depending on the shutter speed. A global shutter camera develops the entire array at the same time. Although Rolling shutter and Global shutter cameras interact with modulation schemes differently, the receivers can still be designed to decode the same transmitted signal.

An OCC link typically has a lower bit rate as compared to that of a system utilizing a photodetector. The bitrate limitation is because the bitrate is dependant on the camera's frame rate. The typical frame rate of 30-60 frames per second (fps) leads to slower camera sampling rates as compared to that of photodiodes. Therefore, OCC links are more suitable for lower bitrate links such as in many IoT applications.

Modulating Retroreflector (MRR): In the late 1990s, the U.S. Naval Research Laboratory (NRL) started conducting experiments on modulating retroreflector (MRR) FSO communication links [109]. An MRR link comprises an interrogator with ample power at one end and a small passive corner cube or a cats eye optical retroreflector coupled to an optical modulator at the other end. The interrogator transmits a beam light beam towards the retroreflector, which modulates and reflects the beam back to the interrogator. The limited duplex MRR link has been successfully used in Terrestrial shore-toshore, boat-to-shore, and sky-to-ground links. Minimal indoor application of the MRR links has been discussed in the literature [110], [111].

\section{Modulation}

Based on the application requirements, a modulation scheme with appropriate transmission reliability, energy, and spectral efficiencies. The most straight forward and commonly used OWC modulation scheme is On-Off keying (OOK) modulation. In OOK, a light beam is switched on and off based on the data bits. Despite the simplicity of OOK, it can be inefficient in high bitrate applications. More sophisticated modulation schemes are needed for high bitrate applications such as Pulse Width Modulation (PWM) [112], Pulse Position Modulation (PPM) or one of its variations, e.g., Variable-PPM (VPM) [97], [113], [114]. The higher efficiency of PPM-based modulation schemes, however, comes at the cost of a more complex timedomain equalization. This additional complexity can pose a challenge for OWC links with low-quality channels [115].

A modulation scheme is either a single-carrier, such as OOK and PPM, or a multi-subcarrier modulation scheme. Singlecarrier modulation schemes have lower bandwidth efficiency, and therefore, at higher bitrates, they experience higher intersymbol interference (ISI) and become inefficient [116].

Subcarrier Intensity Modulation (SIM) and Multiple SIM (MSIM) such as Wavelength Division Multiplexing (WDM) and Orthogonal Frequency-Division Multiplexing (OFDM) offer better bandwidth efficiencies and can help mitigate OWC channel impairments [117]. In SIM-based modulation schemes, a pre-modulated RF signal drives the optical source. A DC bias is added to the pre-modulated signal to obtain an all positive amplitude [118].

More subcarriers must be used to achieve a higher bitrate. However, as the number of subcarriers increases, so does 


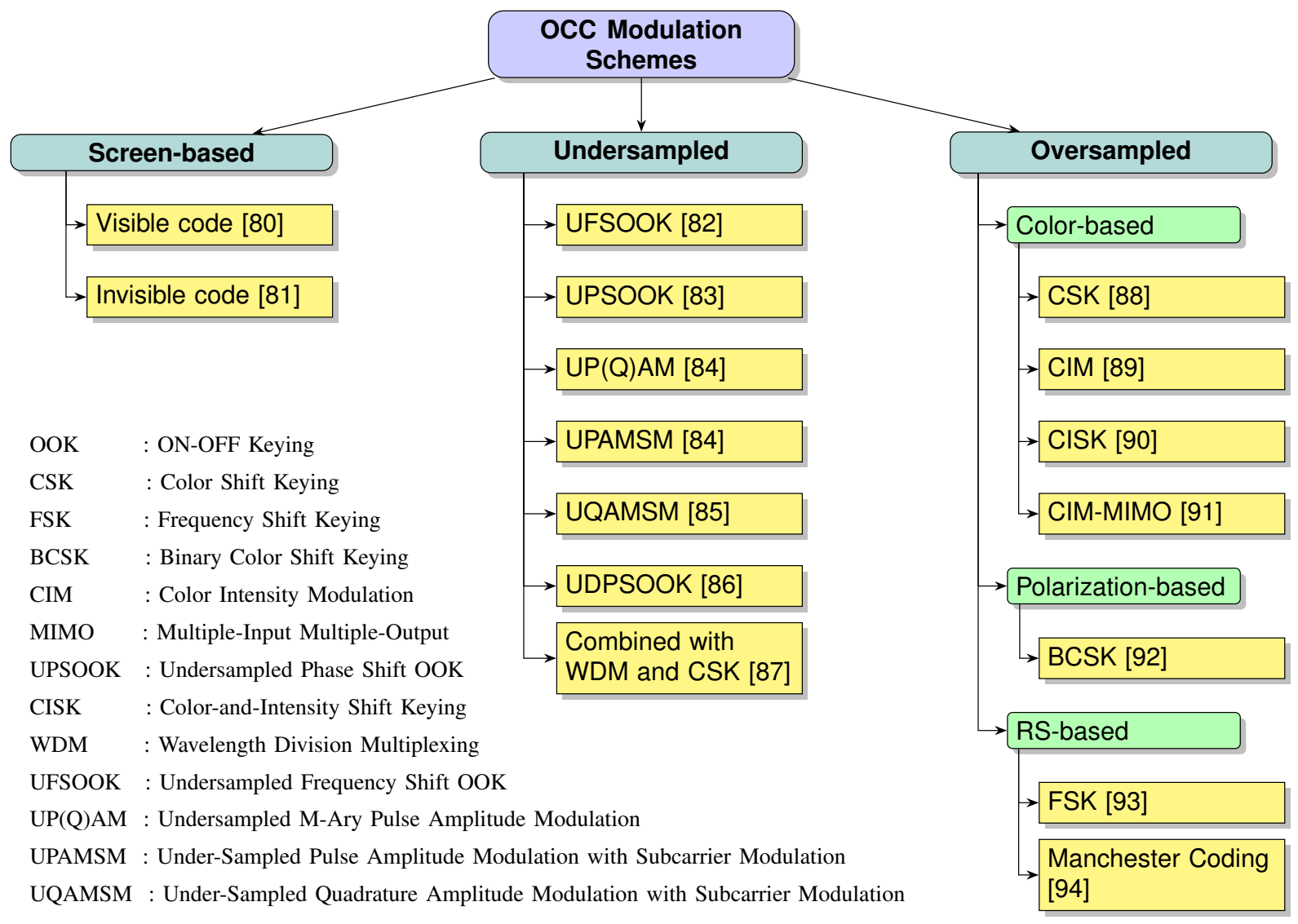

Fig. 8: Summary of main OCC modulation scheme categories and examples

the required non-information DC bias, which can lead to a higher peak-to-average power ratio (PAPR) and worsens the power efficiency [117]. PAPR can be mitigated using reduction techniques and pre-distortion or post-distortion nonlinearitiescompensation methods [119]-[121]. Moreover, the light source nonlinearity causes interference among the subcarriers and broadening of the signal spectrum resulting in mixed signals and Inter-Modulation Distortion (IMD) [118], [122]. The effect of the nonlinearity can be mitigated by using fewer subcarriers at the cost of reduced bitrate, or utilizing spatial diversity, with increases to overall system cost [123].

We refer readers, interested in SIM modulation techniques, to the survey conducted by Hassan et al. in which they discuss the advantages and challenges of SIM/MSIM [117].

Although most of the modulation schemes discussed above can be used for OCC links, most of these modulation schemes modulate faster than the camera frame rate. If the modulation frequency was reduced to be closer to the frame rate, the frequency would be less than the critical flicker frequency (CFF) of the human eye. Any human will be able to distinguish this flickering visibly, making the OCC link inconvenient. Since the camera cannot receive the data as quickly as PD receivers, new schemes that are specific to rolling shutter cameras or global shutter cameras were created [103], [104], [124].

In [103], Luo et al. classify OCC modulations into three categories, namely, screen-based, oversampled, and undersampled modulation schemes. In screen-based modulation schemes, a visible (QR-like) or invisible (embedded) code is used to modulate a 2D array of LEDs. In oversampled modulation techniques, the received signal is oversampled during the demodulation. In an undersampled modulation, which is a form of SIM, a signal is transformed from baseband to passband and then used to modulate light source at a frequency higher than the CFF. The three modulation categories can achieve flickerfree modulation; however, undersampled modulation schemes are more suitable for low data rate, long-range transmission, oversampled schemes are high data rate, but shorter distance. Screen-based modulation can achieve similar data rates as an array of transmitters using undersampled schemes.

We include Figure 8 to depict and familiarize the reader with the different existing OCC modulation categories and schemes. The details of different modulation schemes, however, are beyond the scope of this survey paper. Therefore, we refer interested readers to the recent comprehensive surveys on OCC modulation schemes [103], [104], [124]. Nonetheless, as we review the papers from the literature in Section IV, and if the details of a specific modulation scheme are deemed necessary to understand the paper under consideration, we will provide the reader the details of that specific modulation scheme.

\section{E. Standards}

The ever increasing interest in OWC technology has motivated several initiatives to standardize the technology (see 
TABLE IV: Summary of Existing OWC Standards and Recommendations

\begin{tabular}{|c|c|c|c|c|}
\hline Standard/Recommendation Name & Type & Environment & Status & Focus \\
\hline JEITA CP 1221-1223 [126] & Standard & Indoor & Active & VLC \\
\hline IEEE 802.11 [127] & Standard & Indoor & IR Suspended (1997) & LAN RF and IR Links \\
\hline IEEE 802.11bb [128] & Project & Indoor & Active & $\begin{array}{l}\text { IEEE } 802.11 \text { MAC } \\
\text { Changes to use } \\
\text { Light as a Medium }\end{array}$ \\
\hline IEEE 802.15.7-2018 [129], [130] & Standard & Indoor \& Terrestrial & Active & Short-Range OWC \\
\hline IEEE $802.15 .7 \mathrm{~m}$ [131] & Standard & Indoor \& Terrestrial & Active & OCC \\
\hline ITU G.VLC [132] & Standard & Indoor & Active & $\begin{array}{c}\text { High-Speed VLC } \\
\text { Transceiver }\end{array}$ \\
\hline IOAG.T.OLSG.2012.V1 [135] & Recommendation & Space & Active & $\begin{array}{l}\text { Inter-organization } \\
\text { Standard for Space } \\
\text { FSO Communication }\end{array}$ \\
\hline
\end{tabular}

Table IV). Among the four environments comes the Indoor environment with the most significant standardization efforts, followed by Terrestrial, then Space, and complete absence of standardization in the Underwater environment [13]. We believe that understanding the existing OWC standardization efforts will help us better understand the papers to be reviewed in Section IV. We focus our discussion on short and mediumrange indoor and terrestrial OWC systems.

1) IEEE 802.11: In 1997, IEEE released the standard IEEE 802.11 in which two data links, 1 and $2 \mathrm{Mbps}$, are specified [127]. The first link operates in the $2.4 \mathrm{GHz}$ frequency in the Industrial, Scientific, and Medical (ISM) band, which is now known as the WiFi. The second link operates in the IR band $(850-950 \mathrm{~nm})$ and has a range of $10 \mathrm{~m}$. Two modulation schemes, 16 and 4 PPM, are used for the two data rates 1 and 2 Mbps, respectively. The limitation of the enabling technologies in 1997 prevented the broad deployment of the IR link, and the development of the standard for the IR link was suspended.

2) IEEE 802.15.7 and IEEE 802.15.7m: In 2011, the IEEE 802.15.7-2011 standard for VLC was released, defining the PHY and MAC layers for short-range OWC using VL for Local and metropolitan area networks [13]. In 2014, the ShortRange Optical Wireless Communications Task Group was tasked to revise the IEEE 802.15.7-2011 to broaden the scope of the standard to include IR and near UV as well as including other communication techniques such as OCC. As a result, the revision IEEE 802.15.7-2018 was released in 2018. The standard defines three classes of VLC devices:

- Infrastructure: Also called coordinator is a stationary device that has an unconstrained form factor and ample power supply. The light source is intense and has short and long coverage with high or low data rates.

- Mobile: Movable devices with limited power supply and a constrained form factor. Mobile devices use weak light sources, and thus operates at short ranges and can transmit at high data rates.

- Vehicle: Mobile devices with an unconstrained form factor and moderate power supply. Employs an intense light source to communicate over long distances at low data rates.

The three VLC devices can be arranged in one of three network topologies:

- Star: Supports communication between several devices and one coordinator assigned a unique optical wireless personal area network (OWPAN) identifier not used within the coverage area.

- Peer-to-peer: Supports communication between two close devices, one of which acts as the coordinator.

- Broadcast: Uni-directional transmission from a coordinator to one or more devices without forming a network.

Three PHY layers were defined in the 2011 version of the standard such that they can co-exist but not interoperate. The revised version of the standard slightly modified the original three PHY types and added three new PHY types with a list of modulations schemes for each new PHY type.

- PHY I: Low data rate outdoor applications (11.6 to 266.6 kbps). Employs OOK and VPPM.

- PHY II: Moderate data rate indoor applications (data rate in the tens of Mbps). Employs OOK and VPPM.

- PHY III: Designed to support systems with multiple light sources/detectors at different frequencies (colors). Employs Color-Shift Keying (CSK) with data rate in the tens of Mbps.

- PHY IV: For the use of discrete light sources with data rates $\leq 22 \mathrm{kbps}$. 
TABLE V: Overview of Recent OWC in IoT Research Efforts Based on IoT Domain and Transmission-Receiver Pair

\begin{tabular}{|c|c|c|c|c|c|c|c|}
\hline Domain & $\begin{array}{c}\text { Number of } \\
\text { Publications }\end{array}$ & LD/PD & LED-ID/PD & LED-ID/OCC & LED/PD & LED/OCC & LED/PD-S \\
\hline \hline Healthcare & 9 & NA & NA & NA & {$[136]-[141]$} & {$[142]-[144]$} & NA \\
\hline Smart City & 19 & {$[145]$} & NA & NA & $\begin{array}{c}{[146]-[151]} \\
{[158]-[162]}\end{array}$ & {$[147],[152]-[156]$} & {$[157]$} \\
\hline Commercial & 5 & NA & {$[147],[163]$} & NA & {$[147]$} & {$[147]$} & NA \\
\hline Industrial & 4 & NA & NA & {$[164]$} & {$[165]-[167]$} & NA & NA \\
\hline
\end{tabular}

- PHY V: For diffused surface light sources with data rates $\leq 5.71 \mathrm{kbps}$.

- PHY VI: For video displays with data rates in kbps.

The IEEE $802.15 .7 \mathrm{~m}$ TG focused on the OCC operation which uses PHY IV, V, and VI. The IEEE 802.15.7-2018 standard includes tables listing different operating modes for OCC using different PHY types. For each mode, a modulation scheme, optical clock rate, Forward Error Correction (FEC) scheme, and achievable data rate are also listed.

3) IEEE 802.11 bb: In July 2017, the Light Communication (LC) Topic Interest Group (TIG) was created within the IEEE 802.11 Working Group (WG) to explore the viability of utilizing the light as a medium for wireless communications. As a result of the LC TIG's efforts, an LC Study Group was formed, which worked on developing the Project Authorization Request (PAR) and Criteria for Standards Development (CSD). The IEEE 802.11 WG approved the PAR and CSD in March 2018, followed by the IEEE Standards Association's Standards Board in May 2018, and the new Light Communications amendment to the IEEE Std. 802.11 was created.

The IEEE $802.11 \mathrm{bb}$ LC Task Group on Light Communications focuses on introducing necessary changes to the MAC of the base IEEE 802.11 Stds and reuse of associated services to enable communications using light. Moreover, new PHY mechanisms will be defined to operate using a wide range of light sources developed by different vendors and operating at different modulation bandwidths. The project aims to standardize communication links operating in the range of $380-5,000 \mathrm{~nm}$ with a single-link throughput of $10 \mathrm{Mbps}$ and at least one mode of operation of at least 5 Gbps. Security issues related to the interoperability of the new LC PHY and the existing 802.11 PHYs as a device transitions from one PHY to another will be considered.

4) IEEE 802.15.13: This standard defines a PHY and MAC layer using light wavelengths from $10 \mu \mathrm{m}$ to $190 \mathrm{~nm}$. The standard aims to develop links capable of achieving data rates up to $10 \mathrm{Gbps}$ at distances in the range of $200 \mathrm{~m}$ with unobstructed LOS under varying channel conditions. The standard considers point-to-point, coordinated, and noncoordinated point to multi-point communication links. The coordinated point to multi-point communication is similar to a base station and users in cellular communication. The connectivity must be maintained as a device is moving within the coverage area of a coordinator, and handover as it moves from one coordinator's coverage area to the coverage area of an adjacent coordinator.

\section{F. Putting it All Together}

Pairing a transmitter (LD or LED) and a receiver (PD, PD-S, camera) can lead to different OWC links with varying properties and performance. Using the proper modulation scheme can also help improve the bitrate of the link. With standards being developed, complete networking OWC solutions can be realized.

One of the existing OWC networking solutions is $\mathrm{LiFi}$ technology. LiFi is a high-speed bidirectional local area OWC access network that operates in the VL and IR spectrum bands and builds on the VLC technology [23], [168]. In a LiFi network, LED light fixtures are used for illumination and data communication, simultaneously [169], [170]. This is achieved by equipping each LED with a LiFi chip or modem making the LED fixture an Access Point (AP).

Communication links in LiFi networks are full-duplex (bidirectional). However, several LiFi systems only include a unidirectional OWC link. Typically this involves a stationary infrastructure transmitter such as an overhead light fixture and a user equipment (UE) that utilizes RF to communicate back to the overhead LED and with other devices in the network [151]. This hybrid receiver may be to lower alignment requirements on the user's device as RF requires less attention to transmitter orientation. Other reasons may include the specific hardware required for OWC transmission in a user's device. The device would require a powerful enough transmitter that utilizes a band outside of visible light to prevent user discomfort. Nevertheless, several examples are found of mobile devices capable of duplex LiFi that used the IR band for the uplink channel [141], [150], or where the high rate transmission of OWC in the VL band made bidirectional LiFi desirable [147]. An experiment featuring a $4.5 \mathrm{~mW}$ LED in which data at a speed of 1.1 Gbps was successfully transmitted over a distance of $10 \mathrm{~m}$ [168]. If IR is used for the uplink from the UE, the AP must be equipped with an IR receiver. As the LiFi technology continues to develop, it is being designed to comply with the IEEE $802.11 \mathrm{bb}$ and ITU G.VLC standards [168].

\section{SuRvey OF OWC TEChNOLOGY IN IOT}

In this section, we review and summarize papers in the domain of OWC in IoT.

Table $\mathrm{V}$ gives an overview of the recent major research performed in the domain classified based on the IoT domain on one dimension, and the six possible transmission/receiver pairs on the other dimension. The table also shows the number of publications in each domain. It is clear that the domain 
of Smart Cities is leading the OWC in IoT research. An extended and more detailed chronological summary of the major OWC in IoT studies is tabulated in Table VI. We breakdown this section into subsections based on application domains. Highlighted boxes in Figure 6 represent the areas in which we found OWC-related papers. Therefore, the following subsections will only focus on the healthcare, smart city, and commercial domains.

It is worth pointing that, we distinguish between papers related to general OWC in IoT aspects, and general OWC papers. There is a large number of papers that focus on improving the performance of OWC links in general and not in the specific context of IoT. Although developed approaches can eventually be used in the IoT domains, we believe that surveys focusing on OWC technology are more appropriate for such papers and we consider them out of the scope of our survey. A few examples of such papers are papers focused on LED-ID transmission that did not include an application, but instead gave implementations for localizing a receiver without IoT application context [171]-[175]. Other papers discussing LED/PD implementations that showcase transmission speeds and link distance, but did not extend to application of the link beyond showing connectivity [176]-[179].

\section{A. Healthcare}

The earliest example of an LED/PD OWC link in IoT is the work presented by Miller et al. [136]. In this work, IR-LEDs are utilized to transmit data to and from a ventricular heart assistance device through an implanted PD. The application is capable of maintaining an error-free link of 9600 bps at $150 \mathrm{~mm}$. This paper's main focus was to have the LED/PD transceiver implanted in the patient to allow a wireless link with the heart assistance device.

Using an LED/PD link Abita and Schneider created a link that sent data through porcine skin samples at 1 Mbps [137]. They used an IR LED and PD transceiver to send and receive data with an Active Medical Implant (AMI) at a distance of $24 \mathrm{~mm}$. The AMI can be used in many different situations and can allow care providers to receive patient health information after the AMI is implanted quickly. The authors did not discuss higher-level applications, such as aggregating patient data over time. However, the AMI devices used were capable of storing up to $512 \mathrm{~Kb}$ so that longer-term data could be given to the care provider or wearer regularly.

Another example of transcutaneous LED/PD communication was found in [138]. In this paper, Okamoto et al. tested both IR and VL LEDs. They found that both IR and VL were able to transmit unhindered at $9600 \mathrm{bps}$, but the IR LED transmitted without error at a distance of $45 \mathrm{~mm}$ while the VL-LED was capable of transmitting error-free up to $20 \mathrm{~mm}$.

In [142], Rachim et al. created an experimental LED/OCC setup for transmitting electroencephalogram (EEG) data. This study used a single 3-watt white LED to transmit over LOS to a smartphone on a tripod stand within the same room. They achieved error-free transmissions with an upload speed of 2.4 Kbps at a distance of 4 meters as shown in Figure 9. They proposed that this type of data transfer may replace common RF

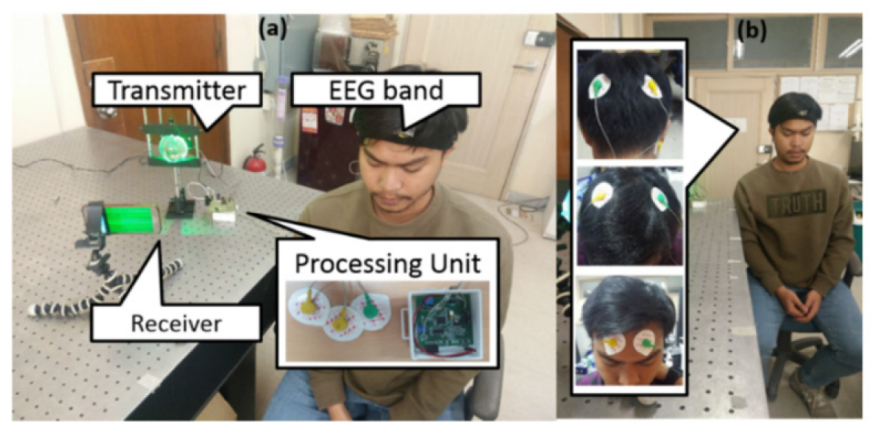

Fig. 9: Experimental setup created by Rachim et al. in [142]

protocols used by wearable devices as a physiologically safer alternative. Even though the authors discussed no application protocol, the transmitted EEG data could be wrapped in an IoT application protocol to enable aggregation and automated healthcare monitoring to further its utility in IoT.

In [139], Dhatchayeny et al. simulated an LED/PD link that sent vital sign measurements to a gateway. The simulation did not mention bit rates or distances but instead focused on Signal to Noise (SNR) ratio versus Bit Error Rates (BER). The team found that they were able to transmit four different signals to the same receiver with a minimal error rate when the SNR value was $12 \mathrm{~dB}$, showing that the MIMO healthcare data aggregation is reliable under the correct conditions.

In another experiment by An et al., an LED/PD link was used to transmit data from multiple electrocardiograms (ECGs) to a custom made dashboard for monitoring the heart health of patients [140]. By using lenses with the LEDs, the authors were able to send data at $40 \mathrm{Kbps}$ at two different distance ranges. The longest distance with a Packet Error Rate of less than 1 was at $8 \mathrm{~m}$. This implementation explored Time hopping as a way to send multiple signals to the same PD receiver. The authors also considered using RF technology to supplement the link when LOS is broken.

Dhatchayeny et al. expand their previous work in [139] and offer a scenario in [141] for biomedical sensors to transmit via IR LED to reduce crowding of the RF spectrum within hospitals and allow high data rate transmission in RF sensitive environments. They propose and implement an LED/PD link for multiple health sensors to transmit patient biomedical data to a single overhead receiver. This allows multiple patients to be monitored in the same room by using a scheduling scheme for transmissions. An IR LED's choice means that there is no visible light to disturb patients or providers, but retains high data rate, LOS secure communications while not causing RF interference. The real-time information can be monitored by a server and send alerts to providers when coupled with an IoT application protocol. The experimental setup enabled transmission at $1.5 \mathrm{~m}$ at $6.4 \mathrm{Mbps}$, but no IoT Object Abstraction Layer application protocol was tested in the research.

In [144], Hasan et al. simulate a hybrid OWC/RF link system to improve the reliability of the signal from healthcare devices, while lowering the amount of RF transmission. The OWC link simulated is an LED/OCC link, and the main 
TABLE VI: Chronological Order of Major OWC in IoT Studies

\begin{tabular}{|c|c|c|c|c|c|c|c|c|c|c|c|c|}
\hline \multirow{2}{*}{$\begin{array}{c}\text { IoT } \\
\text { Business } \\
\text { Domain }\end{array}$} & \multirow{2}{*}{$\begin{array}{l}\text { IoT Ap- } \\
\text { plication } \\
\text { Layer }\end{array}$} & \multirow[t]{2}{*}{ Year } & \multirow{2}{*}{$\begin{array}{c}\text { Study } \\
\text { Type }\end{array}$} & \multirow{2}{*}{$\begin{array}{l}\text { IoT Mid- } \\
\text { dleware } \\
\text { Layer }\end{array}$} & \multicolumn{8}{|c|}{ IoT Object Abstraction Layer } \\
\hline & & & & & \multicolumn{6}{|c|}{ OWC } & $\mathbf{R F}$ & $\begin{array}{l}\text { Appli- } \\
\text { cation } \\
\text { Layer }\end{array}$ \\
\hline $\begin{array}{c}\text { Healthcare } \\
{[136]}\end{array}$ & $\begin{array}{l}\text { Heart } \\
\text { Moni- } \\
\text { toring }\end{array}$ & 1992 & $\begin{array}{l}\text { Imple- } \\
\text { mentation }\end{array}$ & N/A & $\mathrm{P} 2 \mathrm{P}$ & LED & PD & $9.6 \mathrm{Kbps}$ & $150 \mathrm{~mm}$ & FSK & N/A & N/A \\
\hline $\begin{array}{c}\text { Healthcare } \\
\text { [138] }\end{array}$ & $\begin{array}{l}\text { Heart } \\
\text { Moni- } \\
\text { toring }\end{array}$ & 2005 & $\begin{array}{l}\text { Imple- } \\
\text { mentation }\end{array}$ & N/A & $\mathrm{P} 2 \mathrm{P}$ & LED & PD & $9.6 \mathrm{Kbps}$ & $45 \mathrm{~mm}$ & N/A & N/A & N/A \\
\hline $\begin{array}{c}\text { Smart } \\
\text { City [158] }\end{array}$ & $\begin{array}{c}\text { WSN } \\
\text { Man- } \\
\text { agement }\end{array}$ & 2011 & $\begin{array}{c}\text { Imple- } \\
\text { mentation }\end{array}$ & N/A & Star & LED & PD & $\begin{array}{l}115.2 \\
\text { Kbps }\end{array}$ & $60 \mathrm{~cm}$ & N/A & $\begin{array}{c}\text { Ether- } \\
\text { net/PLC }\end{array}$ & N/A \\
\hline $\begin{array}{c}\text { Smart } \\
\text { City [160] }\end{array}$ & $\begin{array}{c}\text { Automated } \\
\text { Lighting }\end{array}$ & 2015 & Simulation & N/A & Star & LED & PD & $1 \mathrm{Kbps}$ & $1 \mathrm{~m}$ & $\begin{array}{c}\text { OOK } \\
\text { Manch- } \\
\text { ester }\end{array}$ & N/A & N/A \\
\hline $\begin{array}{c}\text { Smart } \\
\text { City [152] }\end{array}$ & $\begin{array}{l}\text { Tem- } \\
\text { perature } \\
\text { Moni- } \\
\text { toring }\end{array}$ & 2016 & $\begin{array}{l}\text { Imple- } \\
\text { mentation }\end{array}$ & N/A & Broadcast & LED & $\mathrm{OCC}$ & $150 \mathrm{bps}$ & $12 \mathrm{~m}$ & UOOK & N/A & N/A \\
\hline $\begin{array}{c}\text { Smart } \\
\text { City [146] }\end{array}$ & $\begin{array}{l}\text { Airport } \\
\text { Commu- } \\
\text { nication }\end{array}$ & 2016 & $\begin{array}{l}\text { Theo- } \\
\text { retical }\end{array}$ & $\begin{array}{l}\text { Data } \\
\text { Comm }\end{array}$ & $\mathrm{P} 2 \mathrm{P}$ & LED/LD & PD & N/A & N/A & N/A & Wireless & N/A \\
\hline $\begin{array}{c}\text { Healthcare } \\
{[142]}\end{array}$ & $\begin{array}{l}\text { Wearable } \\
\text { Moni- } \\
\text { toring }\end{array}$ & 2017 & $\begin{array}{l}\text { Imple- } \\
\text { mentation }\end{array}$ & N/A & Broadcast & LED & $\mathrm{OCC}$ & $2.4 \mathrm{Kbps}$ & $4.5 \mathrm{~m}$ & OOK & N/A & N/A \\
\hline $\begin{array}{c}\text { Smart } \\
\text { City [161] }\end{array}$ & $\begin{array}{l}\text { Vehicle } \\
\text { Identi- } \\
\text { fication }\end{array}$ & 2017 & $\begin{array}{c}\text { Imple- } \\
\text { mentation }\end{array}$ & N/A & Star & LED & PD & $\begin{array}{c}\text { DL: } 5 \\
\text { Kbps UL: } \\
1 \text { Kbps }\end{array}$ & N/A & N/A & N/A & N/A \\
\hline $\begin{array}{c}\text { Smart } \\
\text { City [161] }\end{array}$ & $\begin{array}{l}\text { Parking } \\
\text { Detection }\end{array}$ & 2017 & $\begin{array}{l}\text { Imple- } \\
\text { mentation }\end{array}$ & N/A & Star & LED & PD & $\begin{array}{c}\text { DL: } 5 \\
\text { Kbps UL: } \\
1 \mathrm{Kbps}\end{array}$ & N/A & N/A & N/A & N/A \\
\hline $\begin{array}{c}\text { Smart } \\
\text { City [162] }\end{array}$ & $\begin{array}{c}\text { Building } \\
\text { Man- } \\
\text { agement }\end{array}$ & 2017 & $\begin{array}{l}\text { Imple- } \\
\text { mentation }\end{array}$ & N/A & Star & LED & PD & $1 \mathrm{Kbps}$ & $3 \mathrm{~m}$ & N/A & RF/PLC & N/A \\
\hline $\begin{array}{c}\text { Smart } \\
\text { City [153] }\end{array}$ & $\begin{array}{l}\text { Tem- } \\
\text { perature } \\
\text { Moni- } \\
\text { toring }\end{array}$ & 2018 & $\begin{array}{l}\text { Imple- } \\
\text { mentation }\end{array}$ & N/A & Broadcast & LED & OCC & N/A & $4 \mathrm{~m}$ & N/A & N/A & Hadoop \\
\hline $\begin{array}{c}\text { Smart } \\
\text { City [147] }\end{array}$ & $\begin{array}{c}\text { Office } \\
\text { Network }\end{array}$ & 2018 & $\begin{array}{l}\text { Case } \\
\text { Study }\end{array}$ & N/A & Star & LED & $\mathrm{PD}$ & $42 \mathrm{Mbps}$ & $4 \mathrm{~m}$ & N/A & N/A & N/A \\
\hline $\begin{array}{c}\text { Smart } \\
\text { City [147] }\end{array}$ & $\begin{array}{l}\text { Public Lo- } \\
\text { calization }\end{array}$ & 2018 & $\begin{array}{l}\text { Case } \\
\text { Study }\end{array}$ & N/A & $\mathrm{P} 2 \mathrm{P}$ & LED & PD & $1 \mathrm{Mbps}$ & $4 \mathrm{~m}$ & N/A & N/A & N/A \\
\hline $\begin{array}{c}\text { Smart } \\
\text { City [148] }\end{array}$ & $\begin{array}{c}\text { Ambient } \\
\text { Light } \\
\text { Sensing }\end{array}$ & 2018 & $\begin{array}{l}\text { Imple- } \\
\text { mentation }\end{array}$ & N/A & Broadcast & LED & $\mathrm{PD}$ & $120 \mathrm{bps}$ & N/A & OOK & N/A & N/A \\
\hline $\begin{array}{c}\text { Smart } \\
\text { City [149] }\end{array}$ & $\begin{array}{c}\text { Home } \mathrm{Au}- \\
\text { tomation }\end{array}$ & 2018 & $\begin{array}{l}\text { Imple- } \\
\text { mentation }\end{array}$ & $\mathrm{KNX}$ & Broadcast & LED & PD & $5 \mathrm{Kbps}$ & $10 \mathrm{~m}$ & N/A & N/A & N/A \\
\hline
\end{tabular}




\begin{tabular}{|c|c|c|c|c|c|c|c|c|c|c|c|c|}
\hline \multirow{3}{*}{$\begin{array}{c}\text { IoT } \\
\text { Business } \\
\text { Domain }\end{array}$} & \multirow{3}{*}{$\begin{array}{l}\text { IoT Ap- } \\
\text { plication } \\
\text { Layer }\end{array}$} & \multirow[t]{3}{*}{ Year } & \multirow{3}{*}{$\begin{array}{c}\text { Study } \\
\text { Type }\end{array}$} & \multirow{3}{*}{$\begin{array}{c}\text { IoT Mid- } \\
\text { dleware } \\
\text { Layer }\end{array}$} & \multicolumn{8}{|c|}{ IoT Object Abstraction Layer } \\
\hline & & & & & \multicolumn{6}{|c|}{ OWC } & \multirow[t]{2}{*}{$\mathbf{R F}$} & \multirow{2}{*}{$\begin{array}{l}\text { Appli- } \\
\text { cation } \\
\text { Layer }\end{array}$} \\
\hline & & & & & Topology & Tx Type & Rx Type & Data Rate & Distance & Protocol & & \\
\hline $\begin{array}{c}\text { Smart } \\
\text { City }[150]\end{array}$ & $\begin{array}{c}\text { Smart } \\
\text { Vehicle }\end{array}$ & 2018 & $\begin{array}{c}\text { Imple- } \\
\text { mentation }\end{array}$ & N/A & Broadcast & LED & $\mathrm{PD}$ & N/A & $1.5 \mathrm{~m}$ & WBFM & $\begin{array}{c}\text { IEEE } \\
802.110 \mathrm{OB}\end{array}$ & N/A \\
\hline $\begin{array}{c}\text { Smart } \\
\text { City [151] }\end{array}$ & 5G Access & 2018 & Simulation & N/A & Star & LED & $\mathrm{PD}$ & N/A & $3 \mathrm{~m}$ & 16-QAM & N/A & N/A \\
\hline $\begin{array}{c}\text { Smart } \\
\text { City [157] }\end{array}$ & $\begin{array}{l}\text { Building } \\
\text { Automa- } \\
\text { tion }\end{array}$ & 2018 & $\begin{array}{l}\text { Imple- } \\
\text { mentation }\end{array}$ & N/A & Star & LED & PD-S & N/A & N/A & $\begin{array}{l}\text { OOK } \\
\text { Manch- } \\
\text { ester }\end{array}$ & N/A & MQTT \\
\hline $\begin{array}{c}\text { Smart } \\
\text { City [145] }\end{array}$ & $\begin{array}{l}\text { Energy } \\
\text { Conser- } \\
\text { vation }\end{array}$ & 2018 & Simulation & N/A & Broadcast & LD & $\mathrm{PD}$ & $2.5 \mathrm{Gbps}$ & $3 \mathrm{Km}$ & N/A & N/A & N/A \\
\hline $\begin{array}{c}\text { Com- } \\
\text { mercial } \\
{[147]}\end{array}$ & $\begin{array}{c}\text { Smart } \\
\text { Retail } \\
\text { Aswaaq }\end{array}$ & 2018 & $\begin{array}{l}\text { Case } \\
\text { Study }\end{array}$ & N/A & Broadcast & LED & $\mathrm{OCC}$ & N/A & N/A & N/A & N/A & N/A \\
\hline $\begin{array}{c}\text { Com- } \\
\text { mercial } \\
{[147]}\end{array}$ & $\begin{array}{c}\text { Smart } \\
\text { Retail } \\
\text { Carrefour } \\
\text { Lille }\end{array}$ & 2018 & $\begin{array}{l}\text { Case } \\
\text { Study }\end{array}$ & N/A & Broadcast & LED & $\mathrm{OCC}$ & N/A & N/A & N/A & N/A & N/A \\
\hline $\begin{array}{l}\text { Com- } \\
\text { mercial } \\
{[147]}\end{array}$ & $\begin{array}{c}\text { Smart } \\
\text { Retail E. } \\
\text { Leclerc }\end{array}$ & 2018 & $\begin{array}{l}\text { Case } \\
\text { Study }\end{array}$ & N/A & Star & LED & $\mathrm{PD}$ & $1 \mathrm{Mbps}$ & $4 \mathrm{~m}$ & N/A & N/A & N/A \\
\hline $\begin{array}{c}\text { Com- } \\
\text { mercial } \\
{[147]}\end{array}$ & $\begin{array}{l}\text { Smart } \\
\text { Museum } \\
\text { Grand } \\
\text { Curtius } \\
\end{array}$ & 2018 & $\begin{array}{l}\text { Case } \\
\text { Study }\end{array}$ & N/A & Broadcast & LED-ID & $\mathrm{PD}$ & N/A & N/A & N/A & N/A & N/A \\
\hline $\begin{array}{c}\text { Com- } \\
\text { mercial } \\
{[163]}\end{array}$ & $\begin{array}{c}\text { Smart } \\
\text { Museum }\end{array}$ & 2018 & $\begin{array}{l}\text { Imple- } \\
\text { mentation }\end{array}$ & OneM2M & Broadcast & LED-ID & $\mathrm{PD}$ & N/A & $4 \mathrm{~m}$ & N/A & BLE/Zigbee & N/A \\
\hline $\begin{array}{c}\text { Industrial } \\
{[165]}\end{array}$ & $\begin{array}{l}\text { Mobile } \\
\text { Industrial } \\
\text { VLC }\end{array}$ & 2018 & Simulation & N/A & Broadcast & LED & $\mathrm{PD}$ & N/A & $15 \mathrm{~m}$ & N/A & N/A & N/A \\
\hline $\begin{array}{c}\text { Industrial } \\
{[164]}\end{array}$ & $\begin{array}{c}\text { Smart } \\
\text { Warehouse }\end{array}$ & 2018 & $\begin{array}{c}\text { Imple- } \\
\text { mentation }\end{array}$ & N/A & $\mathrm{P} 2 \mathrm{P}$ & LED & $\mathrm{OCC}$ & 90 bps & N/A & PCM & LoraWAN & N/A \\
\hline $\begin{array}{c}\text { Industrial } \\
{[166]}\end{array}$ & $\begin{array}{c}\text { Smart } \\
\text { Manu- } \\
\text { facturing }\end{array}$ & 2018 & $\begin{array}{c}\text { Imple- } \\
\text { mentation }\end{array}$ & N/A & $\mathrm{P} 2 \mathrm{P}$ & LED & $\mathrm{PD}$ & $1 \mathrm{Mbps}$ & $5 \mathrm{~m}$ & N/A & N/A & N/A \\
\hline $\begin{array}{c}\text { Healthcare } \\
\text { [141] }\end{array}$ & $\begin{array}{l}\text { Patient } \\
\text { Moni- } \\
\text { toring }\end{array}$ & 2019 & $\begin{array}{c}\text { Imple- } \\
\text { mentation }\end{array}$ & $\mathrm{N} / \mathrm{A}$ & Broadcast & LED & $\mathrm{PD}$ & $6.4 \mathrm{Mbps}$ & $1.5 \mathrm{~m}$ & OOK & N/A & N/A \\
\hline $\begin{array}{c}\text { Healthcare } \\
{[143]}\end{array}$ & $\begin{array}{c}\text { Smart } \\
\text { Wearable }\end{array}$ & 2019 & $\begin{array}{c}\text { Imple- } \\
\text { mentation }\end{array}$ & N/A & Broadcast & LED & $\mathrm{OCC}$ & 960 bps & $1 \mathrm{~m}$ & N/A & N/A & N/A \\
\hline $\begin{array}{c}\text { Healthcare } \\
\text { [144] }\end{array}$ & $\begin{array}{l}\text { Patient } \\
\text { Moni- } \\
\text { toring }\end{array}$ & 2019 & Simulation & N/A & Broadcast & LED & $\mathrm{OCC}$ & N/A & $4 \mathrm{~m}$ & N/A & BLE & N/A \\
\hline $\begin{array}{c}\text { Smart } \\
\text { City [154] }\end{array}$ & $\begin{array}{c}\text { V2X } \\
\text { Commu- } \\
\text { nication }\end{array}$ & 2019 & $\begin{array}{l}\text { Imple- } \\
\text { mentation }\end{array}$ & N/A & $\mathrm{P} 2 \mathrm{P}$ & LED & $\mathrm{OCC}$ & Varies & $200 \mathrm{~m}$ & HS-PSK & N/A & N/A \\
\hline $\begin{array}{c}\text { Smart } \\
\text { City [155] }\end{array}$ & $\begin{array}{c}\text { V2X } \\
\text { Commu- } \\
\text { nication }\end{array}$ & 2019 & Simulation & N/A & $\mathrm{P} 2 \mathrm{P}$ & LED & $\mathrm{OCC}$ & N/A & N/A & N/A & N/A & N/A \\
\hline $\begin{array}{c}\text { Industrial } \\
{[167]}\end{array}$ & $\begin{array}{l}\text { Manu- } \\
\text { facturing } \\
\text { Robot } \\
\text { Commu- } \\
\text { nication }\end{array}$ & 2019 & $\begin{array}{c}\text { Imple- } \\
\text { mentation }\end{array}$ & N/A & $\mathrm{P} 2 \mathrm{P}$ & LED & $\mathrm{PD}$ & $150 \mathrm{Mbps}$ & N/A & 64-QAM & N/A & N/A \\
\hline $\begin{array}{c}\text { Smart } \\
\text { City }[156]\end{array}$ & $\begin{array}{l}\text { Tem- } \\
\text { perature } \\
\text { Moni- } \\
\text { toring }\end{array}$ & 2020 & $\begin{array}{c}\text { Imple- } \\
\text { mentation }\end{array}$ & $\begin{array}{l}\text { Things- } \\
\text { board }\end{array}$ & Broadcast & LED & $\mathrm{OCC}$ & 5 bps & $6 \mathrm{~m}$ & UFSOOK & WiFi & MQTT \\
\hline
\end{tabular}


component studied was the reliability of the link. When the OCC link becomes unreliable, the RF channel was utilized instead. The authors found that increasing the number of cameras in the room decreased the need to switch to the RF channel and that the OCC link was viable up to 4 meters.

Dhatchayeny et al. propose an experimental LED/OCC link in [143] that can be used to transmit vital sign data. The main consideration was that the link would have a lower bit error rate (BER) to ensure that critical health data remains accurate over the link. The setup involved a $4 \times 4$ panel of RGB LEDs to transmit 48 bits per frame and a 30 fps rolling shutter camera. The experimental setup demonstrated a low BER value transmission of $1.2 \times 10^{-4}$ with a rate of 960 bps at a distance of $100 \mathrm{~cm}$. The transmissions were directly translated into vital sign data, but an application protocol could be utilized over such a link to enable data aggregation.

\section{B. Smart City}

1) Smart Home, Smart Building: In [158], Lee et al. consider how an LED/PD link may be implemented to manage an indoor wireless sensor network. The authors considered LED lighting uniformly distributed throughout a building connected to Power Line Communication (PLC) to provide a secure downlink for any sensor nodes within a wireless sensor network. In their experiments, the authors used WLEDs to transmit from overhead lights to sensor nodes, while the nodes transmitted back using IRLEDs. The added benefit of PLC is that minimal infrastructure changes can be made to enable LED/PD communication in buildings. This system is a general example of how VLC may be utilized within an indoor environment to increase network efficiency over a simple RF system.

Another application is an indoor lighting system that can adjust building lights depending on the ambient light levels. In [160], Warmerdam et al. simulate an LED lighting system of overhead building lights as VLC gateways that communicate with light sensors using LED/PD links. In this example, gateways are connected through wireless RF technology or ethernet. Sensors use LEDs to send 512 bits of ambient light data at $1 \mathrm{Kbps}$ over 1 meter to the overhead lights, which is then aggregated and informs the self-regulation of the overhead lighting.

In [152], Ong and Chung show the results of an LED/OCC link used to monitor indoor temperature. The system achieved $150 \mathrm{bps}$ at $12 \mathrm{~m}$ using Undersampled OOK paired with color modulation of three different colored LEDs at the transmitter side. The results showed that transmission was viable at up to $15 \mathrm{~m}$ during the day, and extended to $25 \mathrm{~m}$ at night when the ambient light was low.

Mariappan et al. explore Ethernet-VLC, PLC-VLC, and RF-VLC links where all the light sources or signage in a building serve as an access point to the network for IoT end devices [162]. This type of device management system enables lighting infrastructure to integrate into wireless sensor networks to perform Building Management services with LED or LED-ID connections. The authors give some application examples of this technology, such as Heating, ventilation, and air conditioning (HVAC) monitoring, surveillance, power management, building security, and communications. The only requirement is that the end device that performs one of these functions must have a compatible OWC receiver and be within the range of the gateway. This setup works well in industrial or commercial settings where overhead lighting is available, and where the RF spectrum may be constrained if there are too many connected devices throughout the facility.

In [145], Siddique et al. propose to use an LD/PD link to send energy usage data from homes to the power substation in a smart village application to maximize energy efficiency. The simulated outdoor link had a distance of up to $3 \mathrm{~km}$ and was tested for feasibility by finding the effects of atmospheric attenuation and scattering during rainy conditions. The key for transmitting at this distance effectively was to utilize an IR LD because the IR band of OWC is least affected by atmospheric conditions. An LD was required to ensure that the focus of the beam remained consistent for the long distance. The data transmitted using this link can ensure efficient electricity distribution by only sending energy to a home when there is demand, conserving the supply at the power station.

In [157], Reddy et al. create an LED/PD-S link and utilize MQTT to monitor and analyze data from an ambient light sensing device. They successfully obtained the data from the end node then analyzed it using machine learning to predict future ambient light levels. There is no proposed benefit by the authors for using a PD-S receiver over a $\mathrm{PD}$ receiver, but if the device were sufficiently low-powered, it could increase the longevity of a battery power supply. It is proposed in the paper that a similar link and application protocol with different sensor devices can be utilized for Smart Building networks.

Zhou et al. demonstrate a Temperature monitoring system in [153] that connects sensor nodes to the gateway through an LED/OCC link. The research included spreading 1000 sensors across 200 rooms on over ten floors to collect temperature data every minute. The authors utilized a single $120 \mathrm{fps}$ CMOS camera per floor to gather data from multiple LED light sources which transmitted the temperature data. The maximum distance of transmission between the LED and the camera was $4 \mathrm{~m}$, but the rate of packet loss was not reported in this paper.

Roch and Martina give an example of an LED/PD link supplemented with PLC to create a low data rate Ambient Light Sensing system in [148]. This project's focus was to demonstrate a compact, low-cost OWC system with a that can operate as both an LED-ID or low data rate LED source. Roch and Martina utilized a single LED lamp to act as the light sensor by using a filtered photodiode, which helped prevent error when exposed to ambient light or other interfering light sources. It could then transmit the received data to another sensor or a gateway depending on network topology. Their data showed that 100-120 bps uplink was possible with the sensor node. No application layer protocols were specified in the paper. This type of OWC transceiver successfully addresses the problem with having a photodiode next to a light source, which is vital for compact OWC capable devices.

A case study of an LED/PD system capable of full-duplex communication was presented in [147]. The implementation was used in multiple offices to enable a full duplex $42 \mathrm{Mbps}$ 


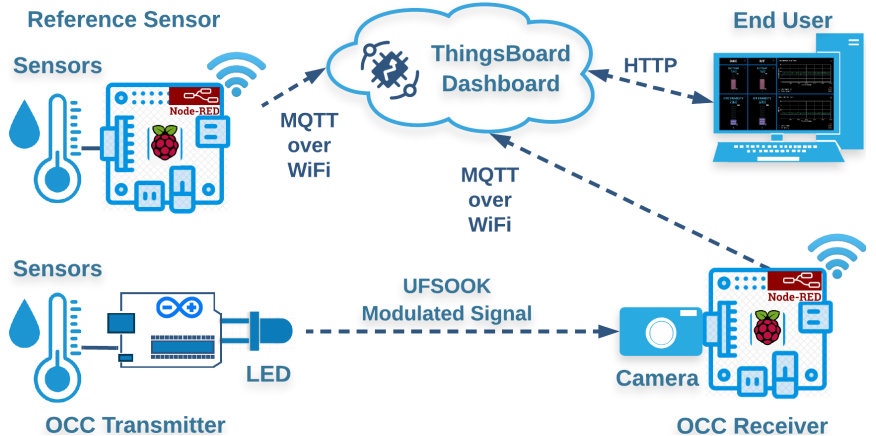

Fig. 10: Figures showing Experimental Setup and Dashboard from [156].

physical link over 4 meters. This system was utilized in a business setting to enable mobile networking with devices but required a USB dongle used as a transceiver to communicate with a gateway. The downlink channel for the end device utilized a visible band while the uplink channel utilized an infrared band to eliminate interference and user discomfort. The link was used to connect office equipment to Ethernet networks, decluttering the RF frequencies, allowing more device connections.

A description of indoor microcells using LED/PD links is presented in [149] where a sensor, transmitter, and overhead light are used to send data about temperature, light, and humidity in one room to a receiver that was connected to a gateway and aggregated the data. This system shows a secure, low power, and practical use of OWC in smart home automation. Sul'aj et al. used KNX as the middleware that managed and controlled devices within the LED generated microcell. This has applications throughout the home and building automation to securely control devices remotely and declutter the RF spectrum in the area.

Using a $5 \mathrm{~mA}$ LED and Raspberry Pi v2 Camera, the authors of [156] created an LED/OCC link, shown in Fig. 10. Sensed ambient temperature and humidity data were transmitted using UFSOOK over a distance of 4 meters to the camera. Then MQTT over WiFi was used to upload to a ThingsBoard dashboard for monitoring in Fig. 11. In 11(b), it shows a test conducted to compare the response time of the OCC link to a WiFi link. A glass of ice was placed near the sensor for a few minutes then removed. It was found that the OCC system lagged by about 5 minutes behind the WiFi link because it was not using real-time demodulation. The link had a maximum bitrate of 5 bps but showed a consistent upload link. This system is another example of how data can be transferred at low power for consistent Smart Home data.

2) Urban Computing: Shen et al. examined the use of OWC in parking infrastructure with LED/PD links to provide three different services [161]. The services included vehicle identification, parking space detection, and positioning. The authors created a scenario where a LED-ID tag is incorporated into each parking spot, and each vehicle using the service has a tag. The parking lot overhead lights act primarily as tag readers, using filtered photodiodes. The overhead light

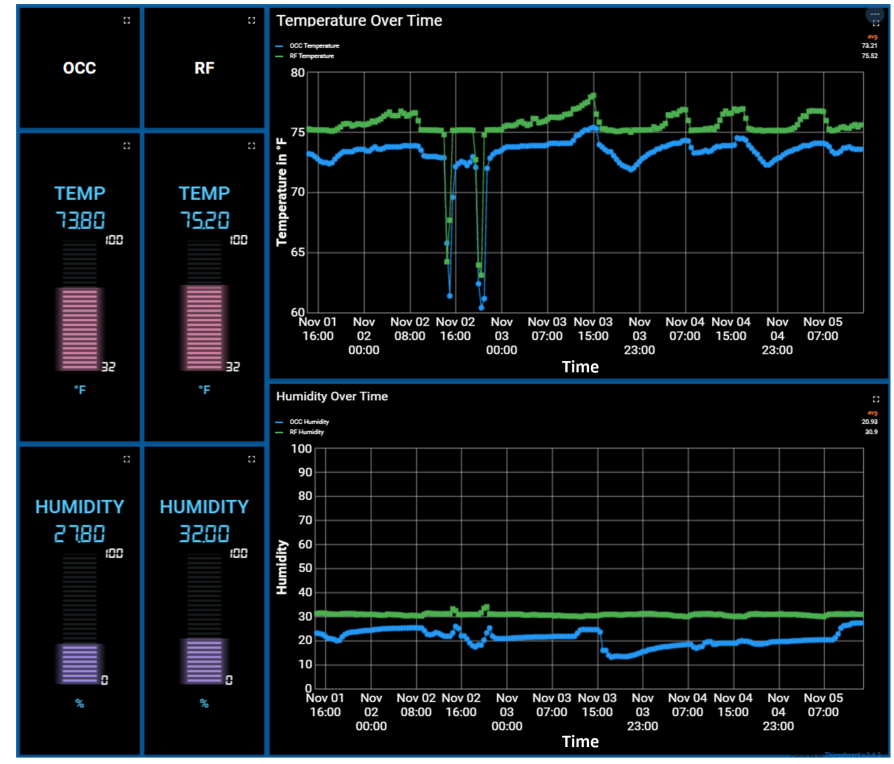

(a)

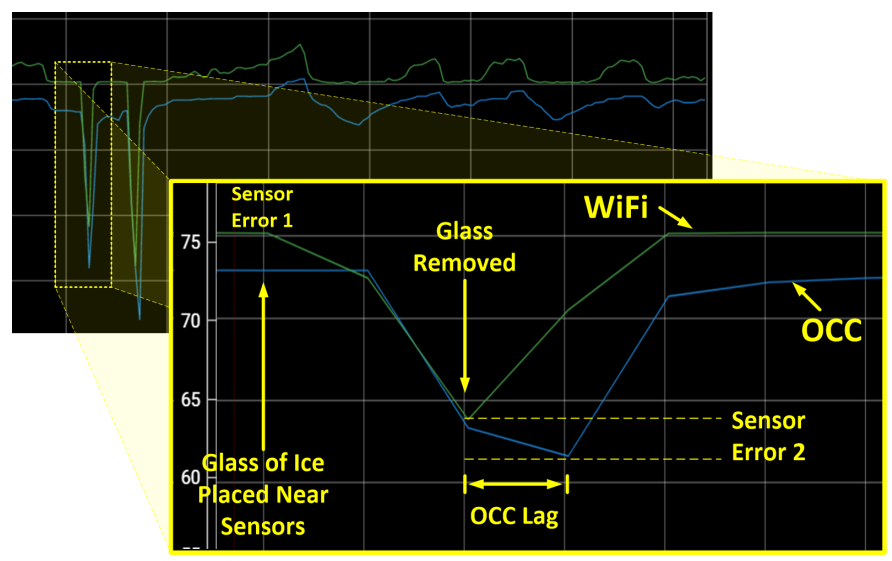

(b)

Fig. 11: ThingsBoard dashboard [156] (a) near real-time temperature and humidity from OCC and WiFi links over a period of five days. (b) Temperature card from the dashboard. The OCC link follows the same trend as the WiFi reference link.

also serves as a transmitter of a carrier signal for the tag that transmits with backscatter from a retro-reflector and LCD shutter combination. The communications from tags on vehicles provide identification of authorized vehicles within the parking lot. Tags placed in parking spots collect data about the lot's occupancy since any tag that is not pinging the receiver is most likely blocked by a vehicle occupying the space. The positioning application is achieved by using an LED-ID/OCC link where ID transmissions from multiple overhead lights send position information to a smart device camera.

Boubakri et al. offer a solution to expand $5 \mathrm{G}$ connectivity within smart cities utilizing VLC gateways in city lighting systems [151]. The authors propose to create LED/PD cells with ubiquitous public lighting and digital signage that are capable of seamless handoff between cells. Through simulations, they examined different handover methods between LED generated cells with a radius of 3 meters that were supposed to represent 
street light gateways. Simulating a nine-cell system showed that up to 60 devices could operate without any blocking, confirming that VLC can assist in 5G connectivity throughout a city. However, since link distance, bit rate, and error rates were not considered, this study only showed the feasibility under indoor conditions.

Another case study from [147], showcased a mobile application developer called Quartier Camille Claudel, which developed a mobile application to receive location information through public lighting. In this case, urban lights were outfitted with Oldecomm modules capable of creating LED/PD or LED/OCC. This technology can form $1 \mathrm{Mbps}$ unidirectional links over 4 meters.

3) Traffic Monitoring: Li et al. implement a prototype of an LED/PD system that utilizes the light emissions from the receiver device to facilitate communication [180]. In this experiment, a tag was equipped with a solar cell to enable independent power, and a retroreflector and LCD shutter pair to enable transmission. The receiver in this communication, referred to as the ViReader, provides the carrier signal for the tag's transmissions through an LED, with which it can also send messages to the tag. The ViReader received messages from the tag through a filtered photodiode. This system was proposed as a solution for low power sensors within indoor environments to communicate without the need for recharging or adding transmission capabilities to roadside signage within a V2I network. The authors found that the tag's transmissions were effective at a rate of $10 \mathrm{Kbps}$ at 2.4 meters.

Delivering Data Comm traffic between ATCs and pilots requires a data communication networking infrastructure. Similar to the application of OWC in vehicular communications, we envision that aircraft-to-aircraft (A2A) and aircraft-to-infrastructure (A2I) can be achieved using T/CC/LOS/\{Medium,Long\} links serving as the infrastructure for Data Comm [146]. We envision that the airport's infrastructure of lights and signages along the taxiways and runways for Data Comm can be utilized. Furthermore, OWC links can be used for aircrafts localization on the airport ground and help raise pilots' situational awareness.

Matus et al. propose a low-cost Vehicle to Infrastructure (V2I) and Vehicle to Vehicle (V2V) solution using LED/PD communications where RF congestion is an issue [150]. An example of V2I is a vehicle communicating with traffic lights through wireless transmissions to indicate that a vehicle is present. $\mathrm{V} 2 \mathrm{~V}$ communication includes communication between vehicles, such as sending data about the distance between them and velocities to enable automated vehicle behavior to prevent a crash. In an experimental setup, they show that LED/PD communications are achievable at 1.5 meters. They propose that this can allow for increased communication efficiency when in a dense RF environment because of the lack of RF interference. Their experiment focused on obtaining velocity and positioning information with the system and found that it was capable of maintaining accurate estimations compared with GPS velocity estimations. A benefit of this system over GPS is that it still operates when the GPS signal is out of range, allowing it to complement RF systems in adverse environments. However, the researchers noted that distance between the link and dynamic environmental factors could significantly degrade the VLC connectivity.

In [154], Thieu et al. experimented with a modulation technique called Hybrid spatial phase-shift keying (HS-PSK) meant to support V2X communications. This modulation scheme attempts to solve problems with LED/OCC links in $\mathrm{V} 2 \mathrm{X}$ applications. The challenges indicated by the authors were the difficulty of automatically detecting the region of interest where transmission is coming from using standard computer vision. The proposed modulation scheme supports private and public communication modes, specifically to pair devices privately while still being able to transmit public information. It also ensures that the camera receivers can demodulate schemes that support dimming, long-distance, very high rate transmissions, and high-mobility transmissions, even if the receiver is low quality. The LED/OCC transmission must also enable both types of camera, global and rolling shutter, to receive the same signal from a variety of transmission sources. During the experimental analysis of the HS-PSK modulation, the authors found that they were able to demonstrate up to 200-meter connections using a pair of $30 \mathrm{~W}$ transmitters.

Hasan et al. proposed a V2X LED/OCC system in [155] that utilizes Convolutional Neural Networks (CNN) for object recognition and tracking. The proposed idea allows the vehicle to recognize traffic signs from images and communicate through OWC simultaneously. The system requires two cameras that are tasked separately, one for object recognition, and the other for receiving OCC signals.

\section{Commercial}

Kim and Koh showed an example of an exhibition service in [163]. In this paper, Kim, and Koh demonstrated a broadcast LED-ID/PD link to mobile receivers. The broadcasting LEDs sent a location specific code that could be referenced by a server for information about the exhibit at the location. The uplink from the mobile receiver to the Aggregation Agent (AA) was through WLAN. The uplink was used to forward the Transmitter ID to the AA, which sent back information about a museum exhibit at the receivers location over 6LoWPAN. This is an example of how LED-ID can be utilized to provide information to receivers within LOS only, like in RFID applications, but with a smaller service area.

In [147], Albraheem et al. propose a number of case studies. The first case study was of implementation of LED/OCC in a supermarket called Carrefour Lille. The application provided location services for products within the store and advertised sales to customers. The data is streamed by overhead lighting in the store and can be received by an OCC application running on customer smartphones. No metrics were discussed within the paper about this particular link.

In another case study, [147] E. Leclerc retail stores utilized indoor positioning to monitor shopping behavior and direct customers to products. The setup utilized Oledcomm's GeoLiFi tracker, a $1 \mathrm{Mbps}$ LED/PD, or LED/OCC link. The customers also received customized notifications and coupons based on their shopping habits. Although data aggregation was used in this example, the architecture was not described. 
The Grand Curtius Museum is another place where Oldecomm's link technology was utilized. From the description given in [147], the system forms an LED-ID/PD link that sends a location tag to an application that uses it to show the museum visitor relevant information about an exhibit. The specifications of the link were not available within the paper.

The last case study from [147] included another example of LED/OCC links that provided customers with promotions, localization, and shopping history within the Aswaaq store in Dubai. Philips developed this application, and no specifications about the link were mentioned.

\section{Industrial}

In an attempt to assess the challenges facing the deployment of VLC in the industrial environment, Almadani et al. propose a modified Monte-Carlo method to investigate the VLC channel characteristics in warehouses [165]. In their proposed work, the authors study different factors that can impact the performance of VLC, such as ceiling heights, receiver's mobility, and illumination levels under different settings related to fixtures angles and output power. The authors study impulse responses, signal to noise ratio (SNR) and bit error rates (BER) for different simulated scenarios using two different commercial lights fixtures offered by OSRAM and Philips used in industrial settings. Simulation results show adequate SNR levels under 5.6, 10, and 15-meter ceiling heights. When a receiver is mobile and moves along a selected path, the simulation shows a $10.4 \mathrm{~dB}$ drop in the SNR.

In [164], Novak et al. demonstrate a hybrid system in which an LED-ID/OCC link is used to supply metadata about warehouse stock, can be seen in Fig. 12. The LED indicator receives status updates through LoRaWAN and then changes color and metadata depending on the status. This allows a visual cue to warehouse management and supplies additional information when queried through a camera attached to glasses, this was shown in Fig. 13. This reduces the RF network load by utilizing RF channels sporadically, while OWC links are the primary form of communication. An implemented system using this technology could automatically schedule orders to manage warehouse stock.

Berenguer et al. implemented a manufacturing robot communication application with a LED/PD system capable of establishing a 1 Mbps links at 5 meters [166]. The system was shown to be capable of transmitting to six different sensors at the same time, allowing simultaneous communication between many different manufacturing robots or other devices. The authors also propose narrowband LEDs and increased PD surface area as possible solutions to increase the transmission rate to $10 \mathrm{Mbps}$.

Another example from Berenguer et al. used an LED/PD system where six photodiode receivers were placed around the manufacturing robot, which had eight IR LED transmitters [167]. These were used to transmit and receive instructions that were also passed through ethernet connections. Berenguer et al. found that the link was capable of 150 Mbps with low BER. The distance of the transmission was not given in the paper; however, within the figure of the experimental setup, the

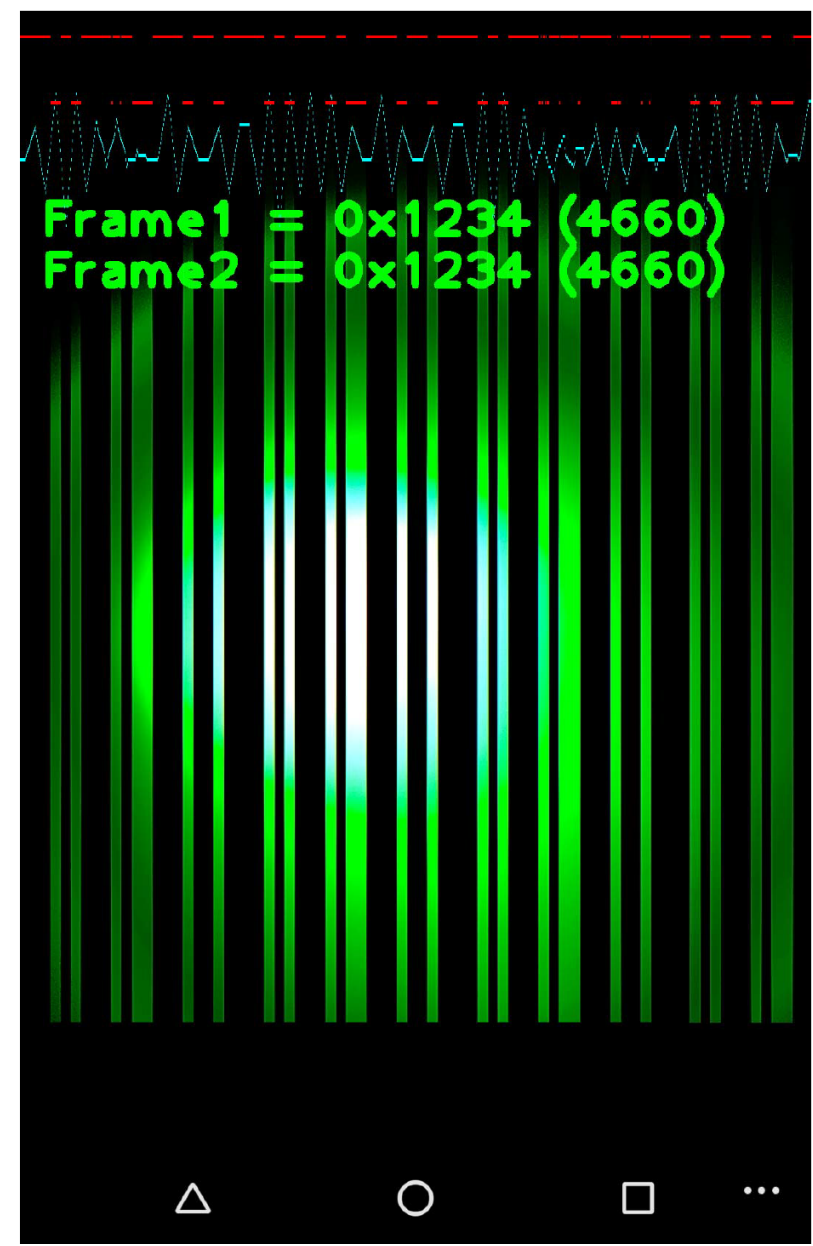

Fig. 12: Logistics Monitoring system from [164] showing the application reading a code from a single transmitter

distance is around one meter from the robot to the receivers. The authors used many transmitters and receivers to ensure that the link would be viable while the robot was able to rotate up to 190 degrees to go through a car manufacturing cycle.

\section{Enabling Technologies: Open Problems and RESEARCH DIRECTIONS}

In this section, we cover some of the related aspects that we found to be applicable for use in IoT but were specifically OWC technologies. These aspects are not domain-related and did not include examples of how they might be used within the context of IoT. We do not cover all possible enabling technologies but provide readers with some of the main aspects that we saw during the literature review.

\section{A. Localization}

Localization is communicating a location and is an important factor within IoT ecosystems. Localization can enable IoT devices to be tracked or to receive data based on a location. For example, multiple broadcast signals from overhead lights can enable a receiver to triangulate its position. Depending on the position, local information can be received, such as an 
User 1

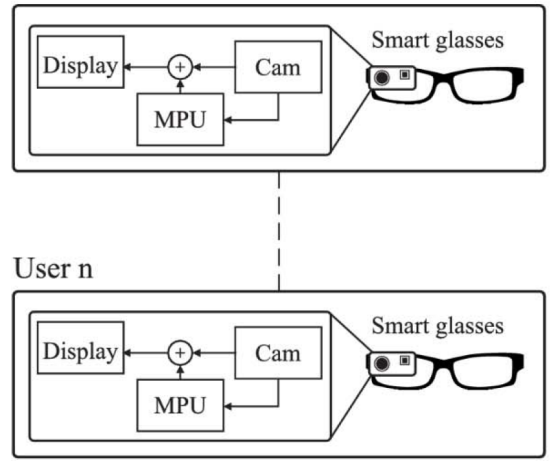

Warehouse

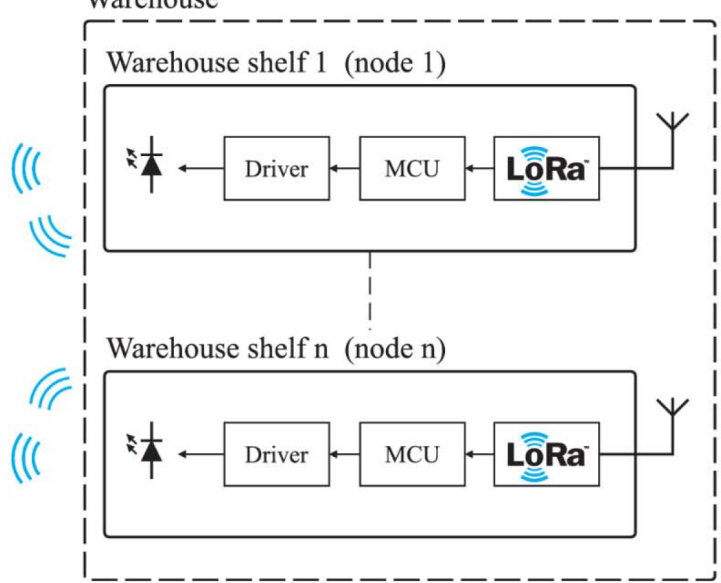

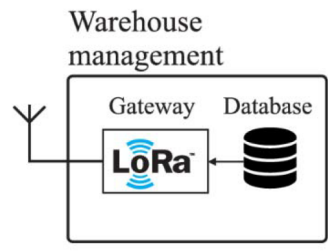

Fig. 13: Logistics Monitoring system from [164] showing the system architecture

advertisement, if the user is inside a store. Because of the much longer wavelengths $(2.4-5 \mathrm{GHz}$ are meters to centimeters long), current RF technologies for localization are not as accurate as Optical localization techniques ( $\mathrm{THz}$ wavelengths are nanometers long). RF localization has resolutions of meters to few centimeters, while OWC localization has resolutions around millimeters or less. There are several examples that we found where OWC can reproduce millimeter or better accuracy for localizing a device [92], [95], [171], [173], [174], [181], [182].

For a better understanding of recent literature surrounding this topic, we also found some surveys that consider VLC solutions for localization [172], [183]. In these surveys, the authors acknowledge that power consumption, security, and throughput are better than RF counterparts and provide additional research done within this field. Within these surveys, there were several common advantages of OWC in localization. First, it can be used for positioning where other positioning services, such as GPS, are not practical due to the line of sight requirements. OWC positioning systems extend positioning services to places that are difficult for satellite radio communication to reach, such as indoors or underground. Another common usage of localization is authentication or security services because of the LOS restriction of light. Authentication codes, connected to specific LED tags, limit the propagation of the sensitive information to line of sight, offering better security than radio communications. Digital Signage is another way that OWC localization can be incorporated within smart commerce and cities. It may allow any lighting to communicate with a mobile device, whether handheld or on a vehicle.

\section{B. Hybrid Networks}

Several researchers have assessed the benefits of a network that uses both RF and OWC physical links. By utilizing a hybrid network, coverage and data rates can be increased as network traffic in the RF spectrum is offloaded to the shorter range, higher data rate VLC cells [184]-[186]. Underwater networks can also benefit from OWC since RF attenuates faster in water [16].

More research opportunities are available to test which IoT applications could use OWC within the network to reduce cost, power usage, increase security, or become useful with OWC supplementing traditional RF communication. The most common implementations of this have been in healthcare applications where OWC is replacing RF communications in equipment typically used in RF sensitive environments.

\section{Device Management}

Some device management protocols have been developed that are specific to using OWC links. This is because the low power consumption needed to transmit optical communications between resource-constrained devices. These new device management protocols allow low data rate communications to signal a device to power on or off the RF transceiver or other sensors to save energy.

For example, in [187] Guo et al. suggest a novel way of synchronizing IoT devices called PSync. The idea is to have receiving devices sample at a low frequency during short wake-up intervals to detect when the synchronization data will arrive from the source. Then wait to turn the receiver back on at the correct time and perform high-frequency sampling to obtain needed synchronization data. They found that PSync consumed roughly one-third of the power as the $2.4 \mathrm{GHz}$ synchronization.

Another example is the IoT Device Management Protocol with Visible Light Communication (IDMP-VLC) [188]. Kim et al. propose using VLC for the uplink channel from IoT devices and a low power RF protocol for downlink. IDMP operates over CoAP to ensure that constrained devices can operate effectively, as CoAP reduces packet length while still being able to integrate with IPv6. 


\section{Hardware}

Several hardware-specific considerations could improve OWC coverage for IoT devices. Retro-reflectors as passive transmitters and Solar cells as receivers were two common ways that were researched to increase low-power device OWC capabilities. The modulation of light coming into a retroreflector is a way for devices to transmit with less power by modulating light from another device with reflectors [180]. Retro-reflectors have a limited transmission distance since they are dependant on external light; the reflection has to be strong enough and directed correctly to overcome the original light source. Creating setups that utilize solar cells to receive both data and power effectively is another factor that could benefit IoT devices [189]. Solar cells cannot modulate as quickly as traditional PD receivers, so the modulation scheme may need to adapt depending on the receiver.

\section{E. Modulation Schemes}

Assessing new OWC modulation schemes and comparing them against established schemes is still an active research area. Modulation schemes are still being tested and modified to maximize data rates and distance depending on the environment and device hardware capabilities [190]. For example, the current OCC schemes are listed in 8 , but there are more schemes in [24] for PD based modulation and Screen-based modulation.

An example of a non-standard modulation scheme called Dark VLC is proposed by Tian et al. in [191], [192]. It requires modulating visible light in very short bursts such that the human eye cannot register it. This method has been shown to have strict distance and data rate limitations but may prove to be an alternative to IR transmission. Kadam and Dhage explored similar methods in [61].

\section{CONClusions}

Optical wireless communication (OWC) is a rapidly developing technology. It gives access to a sizeable unlicensed range of the EM spectrum. Moreover, it has several advantages that make it a suitable wireless technology for future networks and systems such as the Internet of Things (IoT).

In this survey paper, we survey existing research papers in the literature investigating the use of OWC technology in IoT. To this end, we introduce the readers to the concepts and preliminaries of IoT and OWC technologies. We also point the reader to the existing body of existing surveys on each topic. This helps lay the background and explains how and where the OWC technology fits in the IoT architecture.

Using the IoT architecture and the OWC technology elements, we surveyed existing literature combining the two technologies. As a result of our survey, we classified 35 examples of OWC use in IoT systems.

It is found that most of the research done that includes the use of OWC in IoT applications does not consider the IoT's full-stack to observe the effects of OWC on the full system performance. Instead, an OWC link is used in a specific application, and the higher layers of the stack are theorized. Observing how OWC may enable new types of
IoT applications necessitates the creation of full-stack systems because the limitations of OWC may affect the IoT solution under consideration. For example, it can control the protocols chosen, and the design of the middleware, how the user's application interface is built, and inform what type of devices can effectively operate using OWC communication.

To enable applications across expanding IoT domains, the creation of hybrid networks that communicate across both RF and OWC physical links is necessary. There are many RF protocols now used to connect these architectures, but OWC supports certain applications better than current RF protocols. Therefore, OWC can compliment RF networks and increase the bandwidth of systems as more devices become connected.

\section{REFERENCES}

[1] IoT Analytics. (2018) State of the IoT \& Short term outlook 2018. [Online]. Available: https://iot-analytics.com/

[2] Cisco Visual Networking Index (VNI), "Global Mobile Data Traffic Forecast Update, 20172022 White Paper," Cisco, White Paper 1465272001812119, June 2019. [Online]. Available: http://www.cisco.com/c/en/us/solutions/collateral/serviceprovider/visual-networking-index-vni/vni-hyperconnectivity-wp.html

[3] A. Al-Fuqaha et al., "Internet of Things: A Survey on Enabling Technologies, Protocols, and Applications," IEEE Communications Surveys \& Tutorials, vol. 17, no. 4, pp. 2347-2376, 2015.

[4] A.S. Hamza et al., "A Survey on Inter-Cell Interference Coordination Techniques in OFDMA-Based Cellular Networks," IEEE Communications Surveys Tutorials, vol. 15, no. 4, pp. 1642-1670, Fourth 2013.

[5] I.B.F. de Almeida et al., "5G Waveforms for IoT Applications," IEEE Communications Surveys Tutorials, vol. 21, no. 3, pp. 2554-2567, thirdquarter 2019.

[6] FCC. (2019, Jul.) FCC Transforms $2.5 \mathrm{GHz}$ Band for 5G Services. [Online]. Available: https://www.fcc.gov/document/fcc-transforms-25ghz-band-5g-services

[7] FCC. (2019, Jul.) FCC Establishes Procedures for 5G Incentive Auction. [Online]. Available: https://www.fcc.gov/document/fccestablishes-procedures-5g-incentive-auction

[8] F. Gutierrez et al., "On-chip integrated antenna structures in CMOS for $60 \mathrm{GHz}$ WPAN systems," IEEE Journal on Selected Areas in Communications, vol. 27, no. 8, pp. 1367-1378, October 2009.

[9] T.S. Rappaport et al., "Millimeter Wave Mobile Communications for 5G Cellular: It Will Work!" IEEE Access, vol. 1, pp. 335-349, 2013.

[10] M. Agiwal et al., "Next Generation 5G Wireless Networks: A Comprehensive Survey," IEEE Communications Surveys Tutorials, vol. 18 , no. 3, pp. 1617-1655, thirdquarter 2016.

[11] M. Zhang et al., "Will TCP Work in mmWave 5G Cellular Networks?" IEEE Communications Magazine, vol. 57, no. 1, pp. 65-71, January 2019.

[12] A.S. Hamza et al., "Wireless Communication in Data Centers: A Survey," IEEE Communications Surveys Tutorials, vol. 18, no. 3, pp. 1572-1595, thirdquarter 2016.

[13] A.S. Hamza et al., "Classification Framework for Free Space Optical Communication Links and Systems," IEEE Communications Surveys Tutorials, vol. 21, no. 2, pp. 1346-1382, Secondquarter 2019.

[14] M.A. Khalighi and M. Uysal, "Survey on Free Space Optical Communication: A Communication Theory Perspective," IEEE Communications Surveys Tutorials, vol. 16, no. 4, pp. 2231-2258, Fourthquarter 2014.

[15] H. Kaushal and G. Kaddoum, "Optical Communication in Space: Challenges and Mitigation Techniques," IEEE Communications Surveys Tutorials, vol. 19, no. 1, pp. 57-96, Firstquarter 2017.

[16] Z. Zeng et al., "A Survey of Underwater Optical Wireless Communications," IEEE Communications Surveys Tutorials, vol. 19, no. 1, pp. 204-238, Firstquarter 2017.

[17] I. Vasilescu et al., "Data Collection, Storage, and Retrieval with an Underwater Sensor Network," Proceedings of the 3rd International Conference on Embedded Networked Sensor Systems, pp. 154-165, 2005. [Online]. Available: http://doi.acm.org/10.1145/ 1098918.1098936 
[18] M. Dunbabin et al., "Data muling over underwater wireless sensor networks using an autonomous underwater vehicle," Proceedings 2006 IEEE International Conference on Robotics and Automation,ICRA 2006, pp. 2091-2098, May 2006.

[19] D.K. Borah et al., "A review of communication-oriented optical wireless systems," EURASIP Journal on Wireless Communications and Networking, vol. 2012, no. 1, p. 91, Mar 2012. [Online]. Available: https://doi.org/10.1186/1687-1499-2012-91

[20] T. Cogalan and H. Haas, "Why would 5G need optical wireless communications?" 2017 IEEE 28th Annual International Symposium on Personal, Indoor, and Mobile Radio Communications (PIMRC), pp. $1-6$, Oct 2017.

[21] H. Zhang et al., "Energy Efficient Subchannel and Power Allocation for Software-defined Heterogeneous VLC and RF Networks," IEEE Journal on Selected Areas in Communications, vol. 36, no. 3, pp. 658670, March 2018

[22] S.R. Teli et al., "Optical Internet of Things within 5G: Applications and Challenges," 2018 IEEE International Conference on Internet of Things and Intelligence System (IOTAIS), pp. 40-45, Nov 2018.

[23] H. Haas et al., "What is LiFi?" Journal of Lightwave Technology, vol. 34, no. 6, pp. 1533-1544, March 2016.

[24] IEEE, "IEEE Standard for Local and metropolitan area networks-Part 15.7: Short-Range Optical Wireless Communications - Redline," IEEE Std 802.15.7-2018 (Revision of IEEE Std 802.15.7-2011) - Redline, pp. 1-670, April 2019

[25] Z. Wang et al., Visible Light Communications: Modulation and Signal Processing, ser. IEEE Series on Digital \& Mobile Communication. Wiley, 2017. [Online]. Available: https://books.google.com/books?id= AV9CDwAAQBAJ

[26] R. Minerva et al., "Towards a definition of the Internet of Things (IoT)," IEEE IoT Initiative, Tech. Rep. 1, May 2015

[27] L. Atzori et al., "The Internet of Things: A survey," Computer Networks, vol. 54, no. 15, pp. 2787 - 2805, 2010.

[28] D. Uckelmann et al., Architecting the Internet of Things, 1st ed. Springer Publishing Company, Incorporated, 2011.

[29] Miao Wu et al., "Research on the architecture of Internet of Things," 2010 3rd International Conference on Advanced Computer Theory and Engineering(ICACTE), vol. 5, pp. V5-484 - V5-487, Aug 2010.

[30] R. Khan et al., "Future Internet: The Internet of Things Architecture, Possible Applications and Key Challenges," 2012 10th International Conference on Frontiers of Information Technology, pp. 257-260, Dec 2012.

[31] S. Li et al., "The internet of things: a survey," Information Systems Frontiers, pp. 243-259, 2015.

[32] B. Dorsemaine et al., "Internet of Things: A Definition Taxonomy," 2015 9th International Conference on Next Generation Mobile Applications, Services and Technologies, pp. 72-77, Sep. 2015.

[33] V. Rozsa et al., "An Application Domain-Based Taxonomy for IoT Sensors," 23rd ISPE INternational Conference on Transdisciplinary Engineering: Crossing Boundaries, vol. 4, pp. 249-258, Oct. 2016.

[34] H. Boyes et al., "The industrial internet of things (iiot): An analysis framework," Computers in Industry, vol. 101, pp. 1 - 12, 2018. [Online]. Available: http://www.sciencedirect.com/science/article/pii/ S0166361517307285

[35] J. Hller et al., "Chapter 5 - M2M and IoT Technology Fundamentals," in From Machine-To-Machine to the Internet of Things, J. Hller et al., Eds. Oxford: Academic Press, 2014, pp. 81-143. [Online]. Available: http://www.sciencedirect.com/science/article/pii/ B978012407684600005X

[36] P. Sethi and S.R. Sarangi, "Internet of Things: Architectures, Protocols, and Applications," Journal of Electrical and Computer Engineering, vol. 2017, no. 9324035, p. 25, 2017.

[37] P.P. Ray, "A survey on Internet of Things architectures," Journal of King Saud University - Computer and Information Sciences, vol. 30, no. 3, pp. 291-319, 2018.

[38] H. Ahmadi et al., "The application of internet of things in healthcare: a systematic literature review and classification," Universal Access in the Information Society, May 2018.

[39] C. Bormann et al., "CoAP: An Application Protocol for Billions of Tiny Internet Nodes," IEEE Internet Computing, vol. 16, no. 2, pp. 62-67, March 2012.

[40] "MQTT Version 5.0," OASIS Standard, Tech. Rep., Mar. 2019. [Online]. Available: https://docs.oasis-open.org/mqtt/mqtt/v5. 0/os/mqtt-v5.0-os.html

[41] P. Saint-Andre, "Extensible Messaging and Presence Protocol (XMPP): Core," Internet Engineering Task Force (IETF), Request for Comments 6120, Mar. 2011.
[42] “OASIS Advanced Message Queuing Protocol(AMQP) Version 1.0," OASIS Standard, Tech. Rep., Oct. 2012. [Online]. Available: http: //docs.oasis-open.org/amqp/core/v1.0/amqp-core-overview-v1.0.html

[43] "Data Distribution Service V. 1.4," Object Management Group (OMG), Tech. Rep., Mar. 2015.

[44] C. Pereira and A. Aguiar, "Towards efficient mobile M2M communications: Survey and open challenges," Sensors, vol. 14, no. 10, pp. 19582-19608, 2014.

[45] V. Karagiannis et al., "A survey on application layer protocols for the internet of things," Transaction on IoT and Cloud computing, vol. 3 , no. 1, pp. 11-17, 2015.

[46] S. Profanter et al., "OPC UA versus ROS, DDS, and MQTT: Performance Evaluation of Industry 4.0 Protocols," 2019 IEEE International Conference on Industrial Technology (ICIT), pp. 955-962, Feb 2019.

[47] Z. Shelby and C. Bormann, 6LoWPAN: The Wireless Embedded Internet. Wiley Telecom, 2009

[48] J. Lee et al., "A Comparative Study of Wireless Protocols: Bluetooth, UWB, ZigBee, and Wi-Fi," IECON 2007 - 33rd Annual Conference of the IEEE Industrial Electronics Society, pp. 46-51, Nov 2007.

[49] F.P. Rezha and S.Y. Shin, "Performance evaluation of ISA100.11A industrial wireless network," IET International Conference on Information and Communications Technologies (IETICT 2013), pp. 587-592, April 2013.

[50] J. Song et al., "WirelessHART: Applying Wireless Technology in RealTime Industrial Process Control," 2008 IEEE Real-Time and Embedded Technology and Applications Symposium, pp. 377-386, April 2008.

[51] P. Weber et al., "IPv6 over LoRaWAN," 2016 3rd International Symposium on Wireless Systems within the Conferences on Intelligent Data Acquisition and Advanced Computing Systems (IDAACS-SWS), pp. 75-79, Sep. 2016.

[52] I.D. Chakeres and E.M. Belding-Royer, "AODV routing protocol implementation design," 24th International Conference on Distributed Computing Systems Workshops, 2004. Proceedings, pp. 698-703, March 2004.

[53] J. Sobral et al., "LOADng-IoT: An Enhanced Routing Protocol for Internet of Things Applications over Low Power Networks," Sensors, vol. 19, no. 1, p. 150, Jan. 2019.

[54] J. Hui and J. Vasseur, "RPL: IPv6 routing protocol for low-power and lossy networks," Internet Requests for Comment, RFC Editor, Fremont, CA, USA, Tech. Rep, vol. 6550, 2012.

[55] T.H. Clausen and A.C. de Verdiere, "The Lightweight on-Demand adhoc Distance-Vector Routing pRotocol-Next Generation (LOADng)," 2011.

[56] P. Asghari et al., "Internet of Things applications: A systematic review," Computer Networks, vol. 148, pp. 241-261, 2019.

[57] S. Bandyopadhyay et al., "Role of middleware for internet of things: A study," International Journal of Computer Science and Engineering Survey, vol. 2, no. 3, pp. 94-105, 2011.

[58] A.M. Reda and S. Khattab, "Semantic Technology in Internet of Things Mashup Platforms," Proceedings of the 3rd Africa and Middle East Conference on Software Engineering, pp. 47-48, 2017. [Online]. Available: https://doi.org/10.1145/3178298.3178309

[59] K. Zhao and L. Ge, "A Survey on the Internet of Things Security," 2013 Ninth International Conference on Computational Intelligence and Security, pp. 663-667, Dec 2013.

[60] L.D. Xu et al., "Internet of Things in Industries: A Survey," IEEE Transactions on Industrial Informatics, vol. 10, no. 4, pp. 2233-2243, Nov 2014.

[61] K. Kadam and M.R. Dhage, "Visible Light Communication for IoT," 2016 2nd International Conference on Applied and Theoretical Computing and Communication Technology (iCATccT), pp. 275-278, July 2016.

[62] H. Arasteh et al., "Iot-based smart cities: A survey," 2016 IEEE 16th International Conference on Environment and Electrical Engineering (EEEIC), pp. 1-6, June 2016.

[63] I. Yaqoob et al., "Internet of Things Architecture: Recent Advances, Taxonomy, Requirements, and Open Challenges," IEEE Wireless Communications, vol. 24, pp. 10-16, 2017.

[64] A.H. Ngu et al., "IoT Middleware: A Survey on Issues and Enabling Technologies," IEEE Internet of Things Journal, vol. 4, no. 1, pp. 1-20, Feb 2017.

[65] J. Hou et al., "A survey on internet of things security from data perspectives," Computer Networks, vol. 148, pp. 295-306, 2019. [Online]. Available: http://www.sciencedirect.com/science/article/pii/ S1389128618306844 
[66] B. Turan et al., Visible Light Communications in Industrial Internet of Things (IIoT). Cham: Springer International Publishing, 2019, pp. 163-191. [Online]. Available: https://doi.org/10.1007/978-3-03024892-5 8

[67] M.Z. Chowdhury et al., "The Role of Optical Wireless Communication Technologies in 5G/6G and IoT Solutions: Prospects, Directions, and Challenges," Applied Sciences, vol. 9, no. 20, p. 4367, Oct 2019. [Online]. Available: http://dx.doi.org/10.3390/app9204367

[68] P.N. Ji, "Optical technologies for the Internet of Things era," Optical Data Storage 2017: From New Materials to New Systems, vol. 10384, pp. 1 - 6, 2017. [Online]. Available: https://doi.org/10.1117/12. 2276426

[69] J. Ding et al., "IoT Connectivity Technologies and Applications: A Survey," IEEE Access, 2020.

[70] A.S. Hamza et al., "Evolution of data centers: A critical analysis of standards and challenges for FSO links," 2015 IEEE Conference on Standards for Communications and Networking (CSCN), pp. 100-105, Oct 2015.

[71] I.E. Lee et al., "Near-Infrared Wireless Optical Communication with Particulates In-Suspension over the Underwater Channel," Conference on Lasers and Electro-Optics, p. STh3O.4, 2017.

[72] I. Alimi et al., "Challenges and Opportunities of Optical Wireless Communication Technologies," in Optical Communication Technology, P. Pinho, Ed. Rijeka: InTech, 2017, ch. 2.

[73] A. Vavoulas et al., "A Survey on Ultraviolet C-Band (UV-C) Communications," IEEE Communications Surveys Tutorials, vol. 21, no. 3, pp. 2111-2133, thirdquarter 2019

[74] P.H. Pathak et al., "Visible Light Communication, Networking, and Sensing: A Survey, Potential and Challenges," IEEE Communications Surveys Tutorials, vol. 17, no. 4, pp. 2047-2077, Fourthquarter 2015.

[75] I. Takai et al., "Optical Vehicle-to-Vehicle Communication System Using LED Transmitter and Camera Receiver," IEEE Photonics Journal, vol. 6, no. 5, pp. 1-14, Oct 2014

[76] M. Kavehrad et al., Short-Range Optical Wireless: Theory and Applications. Wiley, 2015

[77] P.P. Manousiadis et al., "Organic semiconductors for visible light communications," Philosophical Transactions of the Royal Society A: Mathematical, Physical and Engineering Sciences, vol. 378, no. 2169, p. 20190186, 2020. [Online]. Available: https://royalsocietypublishing. org/doi/abs/10.1098/rsta.2019.0186

[78] D. Tsonev et al., "A 3-Gb/s Single-LED OFDM-Based Wireless VLC Link Using a Gallium Nitride $\mu$ LED," IEEE Photonics Technology Letters, vol. 26, no. 7, pp. 637-640, 2014.

[79] C.W. Chen et al., "Visible light communications for the implementation of internet-of-things," Optical Engineering, vol. 55, no. 6, pp. 1-3, 2016. [Online]. Available: https://doi.org/10.1117/1.OE.55.6.060501

[80] R. Boubezari et al., "Smartphone Camera Based Visible Light Communication," Journal of Lightwave Technology, vol. 34, no. 17, pp. 4121-4127, 2016.

[81] V. Nguyen et al., "High-rate flicker-free screen-camera communication with spatially adaptive embedding," in IEEE INFOCOM 2016 - The 35th Annual IEEE International Conference on Computer Communications, 2016, pp. 1-9.

[82] R.D. Roberts, "Undersampled frequency shift ON-OFF keying (UFSOOK) for camera communications (CamCom)," in 2013 22nd Wireless and Optical Communication Conference, 2013, pp. 645-648.

[83] P. Luo et al., "Undersampled phase shift ON-OFF keying for camera communication," in 2014 Sixth International Conference on Wireless Communications and Signal Processing (WCSP), 2014, pp. 1-6.

[84] P. Luo et al., "Undersampled-PAM with subcarrier modulation for camera communications," in 2015 Opto-Electronics and Communications Conference (OECC), 2015, pp. 1-3.

[85] P. Luo et al., "Experimental Demonstration of a 1024-QAM Optical Camera Communication System," IEEE Photonics Technology Letters, vol. 28, no. 2, pp. 139-142, 2016.

[86] N. Liu et al., "Undersampled differential phase shift on-off keying for optical camera communications," Journal of Communications and Information Networks, vol. 2, no. 4, pp. 47-56, Dec. 2017.

[87] P. Luo et al., "Experimental demonstration of rgb led-based optical camera communications," IEEE Photonics Journal, vol. 7, no. 5, pp. $1-12,2015$.

[88] E. Monteiro and S. Hranilovic, "Design and Implementation of Color-Shift Keying for Visible Light Communications," J. Lightwave Technol., vol. 32, no. 10, pp. 2053-2060, May 2014. [Online]. Available: http://jlt.osa.org/abstract.cfm?URI=jlt-32-10-2053

[89] K. Ahn and J.K. Kwon, "Color Intensity Modulation for Multicolored Visible Light Communications," IEEE Photonics Technology Letters, vol. 24 , no. 24 , pp. 2254-2257, 2012
[90] W. Chen et al., "Color-and-Intensity Shift Keying for Visible Light Communication," IEEE Communications Letters, vol. 22, no. 9, pp. 1790-1793, 2018.

[91] W. Huang et al., "Design and implementation of a real-time CIM-MIMO optical camera communication system," Opt. Express, vol. 24, no. 21, pp. 24567-24579, Oct 2016. [Online]. Available: http://www.opticsexpress.org/abstract.cfm?URI=oe-24-21-24567

[92] Z. Yang et al., "Wearables Can Afford: Light-Weight Indoor Positioning with Visible Light," Proceedings of the 13th Annual International Conference on Mobile Systems, Applications, and Services, pp. 317-330, 2015. [Online]. Available: https://doi.org/10.1145/2742647.2742648

[93] N. Rajagopal et al., "Visual light landmarks for mobile devices," IPSN14 Proceedings of the 13th International Symposium on Information Processing in Sensor Networks, pp. 249-260, 2014.

[94] C. Danakis et al., "Using a CMOS camera sensor for visible light communication," 2012 IEEE Globecom Workshops, pp. 1244-1248, 2012.

[95] Y.S. Kuo et al., "Luxapose: Indoor Positioning with Mobile Phones and Visible Light," Proceedings of the 20th Annual International Conference on Mobile Computing and Networking, pp. 447-458, 2014.

[96] J.M. Kahn and J.R. Barry, "Wireless infrared communications," Proc. IEEE, vol. 85, no. 2, pp. 265-298, 1997.

[97] Z. Ghassemlooy et al., Optical Wireless Communications: System and Channel Modelling with MATLAB, 1st ed. Taylor \& Francis, 2012.

[98] D.J.T. Heatley et al., "Optical wireless: The story so far," IEEE Commun. Mag., vol. 36, no. 12, pp. 72-74, 1998.

[99] C.H. Liu et al., "Graphene photodetectors with ultra-broadband and high responsivity at room temperature," Nature Nanotechnology, vol. 9 , pp. 273-278, 2014.

[100] F.H.L. Koppens et al., "Photodetectors based on graphene, other twodimensional materials and hybrid systems," Nature Nanotechnology, vol. 9, pp. 780-793, Oct. 2014.

[101] C. Ferrante et al., "Raman spectroscopy of graphene under ultrafast laser excitation," Nature Communications, vol. 9, no. 308, 2018.

[102] Z. Wang et al., "On the Design of a Solar-Panel Receiver for Optical Wireless Communications With Simultaneous Energy Harvesting," IEEE Journal on Selected Areas in Communications, vol. 33, no. 8 , pp. 1612-1623, 2015.

[103] P. Luo et al., "Undersampled-Based Modulation Schemes for Optical Camera Communications," IEEE Communications Magazine, vol. 56, no. 2, pp. 204-212, 2018

[104] N. Saeed et al., "Optical Camera Communications: Survey, Use Cases, Challenges, and Future Trends," 2018.

[105] N. Saha et al., "Survey on optical camera communications: challenges and opportunities," IET Optoelectronics, vol. 9, no. 5, pp. 172-183, 2015.

[106] J.H. Bae et al., "Smartphone Image Receiver Architecture for Optical Camera Communication," Wireless Personal Communications, vol. 93, no. 4, pp. 1043-1066, Apr 2017. [Online]. Available: https://doi.org/10.1007/s11277-017-3971-3

[107] M.Z. Chowdhury et al., "A Comparative Survey of Optical Wireless Technologies: Architectures and Applications," IEEE Access, vol. 6 , pp. 9819-9840, 2018.

[108] W. Liu and Z. Xu, "Some practical constraints and solutions for optical camera communication," Phil. Trans. R. Soc. A, Mar. 2020.

[109] R. Mahon et al., "Free-space optical communication link at $1550 \mathrm{~nm}$ using multiple-quantum-well modulating retroreflectors in a marine environment," Proc. SPIE, vol. 5160, pp. 456-465, 2004.

[110] W. Noonpakdee, "Indoor optical wireless communications employing corner cube retroreflector for health monitoring system," 2013 Fifth International Conference on Ubiquitous and Future Networks (ICUFN), pp. 674-678, 2013.

[111] W. Noonpakdee, "Performance analysis of passive Active optical wireless transmission for personal health monitoring," 2014 Sixth International Conference on Ubiquitous and Future Networks (ICUFN), pp. 17-21, 2014.

[112] J. Lee et al., "Optical Pulse Width Modulated Multilevel Transmission in CIS-Based VLC," IEEE Photonics Technology Letters, vol. 29, no. 15 , pp. $1257-1260,2017$

[113] A. Sevincer et al., "LIGHTNETs: Smart LIGHTing and Mobile Optical Wireless NETworks A Survey," Communications Surveys Tutorials, IEEE, vol. 15, no. 4, pp. 1620-1641, Fourth 2013.

[114] M.A. Khalighi et al., "Underwater wireless optical communication; recent advances and remaining challenges," Intl. Conf. on Transparent Optical Networks (ICTON), pp. 1-4, July 2014. 
[115] R. Hou et al., "A Brief Survey of Optical Wireless Communication," Australasian Symposium on Parallel and Distributed Computing (AusPDC 2015), vol. 163, pp. 41-50, 2015.

[116] M.S. Islim and H. Haas, "Modulation Techniques for Li-Fi," ZTE Communications, vol. 14, pp. 29-40, 2016.

[117] M.Z. Hassan et al., "Subcarrier Intensity Modulated OpticalWireless Communications: A Survey from Communication Theory Perspective,' ZTE COMMUNICATIONS, April 2016.

[118] A. Bekkali et al., "Transmission Analysis of OFDM-Based Wireless Services Over Turbulent Radio-on-FSO Links Modeled by GammaGamma Distribution," IEEE Photonics Journal, vol. 2, no. 3, pp. 510 520, June 2010.

[119] Z. Dong et al., " $8 \times 9.95-\mathrm{Gb} / \mathrm{s}$ Ultra-Dense WDM-PON on a 12.5 GHz Grid With Digital Pre-Equalization," IEEE Photonics Technology Letters, vol. 25, no. 2, pp. 194-197, Jan 2013.

[120] Z. Yu et al., "Iterative Clipping for PAPR Reduction in Visible Light OFDM Communications," in 2014 IEEE Military Communications Conference, Oct 2014, pp. 1681-1686.

[121] J. Zhang et al., "Time-domain digital pre-equalization for band-limited signals based on receiver-side adaptive equalizers," Opt. Express, vol. 22, no. 17, pp. 20 515-20529, Aug 2014.

[122] D. Tsonev et al., "Complete Modeling of Nonlinear Distortion in OFDM-Based Optical Wireless Communication," Journal of Lightwave Technology, vol. 31, no. 18, pp. 3064-3076, Sept 2013.

[123] J.B. Carruthers and J.M. Kahn, "Multiple-subcarrier modulation for nondirected wireless infrared communication," IEEE Journal on Selected Areas in Communications, vol. 14, no. 3, pp. 538-546, Apr 1996.

[124] D.T. Nguyen et al., "Enhancement of Data Rate and Packet Size in Image Sensor Communications by Employing Constant Power 4 PAM," IEEE Access, vol. 6, pp. 8000-8010, 2018.

[125] (2020) Infrared Data Association. [Online]. Available: https://www. irda.org/

[126] (2020) Visible Light Communications Association. [Online]. Available: http://vlca.net/standard/

[127] R.T. Valadas et al., "The infrared physical layer of the IEEE 802.11 standard for wireless local area networks," IEEE Comm. Mag., vol. 36 , no. 12, pp. 107-112, Dec 1998

[128] (2020) Status of IEEE 802.11 Light Communication TG. [Online]. Available: http://www.ieee802.org/11/Reports/tgbb_update.htm

[129] (2018) 802.15.7-2018 - IEEE Standard for Local and metropolitan area networks-Part 15.7: Short-Range Optical Wireless Communications. [Online]. Available: https://standards.ieee.org/content/ieee-standards/ en/standard/802 15 7-2018.html

[130] "IEEE Standard for Local and metropolitan area networks-Part 15.7: Short-Range Optical Wireless Communications," IEEE Std 802.15.72018 (Revision of IEEE Std 802.15.7-2011), pp. 1-407, 2019.

[131] T. Nguyen et al., "Technical Issues on IEEE 802.15.7m Image Sensor Communication Standardization," IEEE Communications Magazine, vol. 56, no. 2, pp. 213-218, 2018.

[132] (2018) High speed indoor visible light communication transceiver System architecture, physical layer and data link layer specification. [Online]. Available: https://www.itu.int/md/T17-SG15-180129-TDWP1-0203/en

[133] (2017) P802.15.13 - Standard for Multi-Gigabit per Second Optical Wireless Communications (OWC) with Ranges up to 200 meters. [Online]. Available: https://standards.ieee.org/project/802_15_13.html

[134] Fixed service applications using free-space optical links, International Telecommunication Union Std. F.2106-1 (2010).

[135] (2012) Interagency Operations Advisory Group - Optical Link Study Group. [Online]. Available: https://www.ioag.org/PublicDocuments/ OLSGReport_Final_06_05_12.pdf

[136] J.A. Miller et al., "Transcutaneous optical telemetry system for an implantable electrical ventricular heart assist device," Medical and Biological Engineering and Computing, vol. 30, no. 3, pp. 370-372, May 1992. [Online]. Available: https://doi.org/10.1007/BF02446977

[137] J.L. Abita and W. Schneider, "Transdermal Optical Communications," Johns Hopkins Apl Technical Digest, vol. 25, no. 3, pp. 261-268, 2004

[138] E. Okamoto et al., "Development of a bidirectional transcutaneous optical data transmission system for artificial hearts allowing longdistance data communication with low electric power consumption," Journal of Artificial Organs, vol. 8, no. 3, pp. 149-153, Sep 2005. [Online]. Available: https://doi.org/10.1007/s10047-005-0299-7

[139] D.R. Dhatchayeny et al., "A novel optical body area network for transmission of multiple patient vital signs," 2017 Ninth International Conference on Ubiquitous and Future Networks (ICUFN), pp. 542544, July 2017.
[140] J. An et al., "Multiple bio-monitoring system using visible light for electromagnetic-wave free indoor healthcare," Optics Communications, vol. 405, pp. 107 - 113, 2017. [Online]. Available: http://www. sciencedirect.com/science/article/pii/S0030401817306855

[141] D.R. Dhatchayeny et al., "Infrared Based Multiple-Patient Monitoring in Indoor Optical Wireless Healthcare Systems," IEEE Sensors Journal, pp. 1-1, 2019.

[142] V.P. Rachim et al., "Demonstration of long-distance hazard-free wearable EEG monitoring system using mobile phone visible light communication," Optics Express, vol. 25, no. 2, pp. 713-719, Jan 2017. [Online]. Available: http://www.opticsexpress.org/abstract.cfm? URI=oe-25-2-713

[143] D.R. Dhatchayeny and Y.H. Chung, "Optical extra-body communication using smartphone cameras for human vital sign transmission," Appl. Opt., vol. 58, no. 15, pp. 3995-3999, May 2019. [Online]. Available: http://ao.osa.org/abstract.cfm?URI=ao-58-15-3995

[144] M.K. Hasan et al., "Real-Time Healthcare Data Transmission for Remote Patient Monitoring in Patch-Based Hybrid OCC/BLE Networks," Sensors, vol. 19, no. 5, 2019. [Online]. Available: https://www.mdpi.com/1424-8220/19/5/1208

[145] A.R.M. Siddique and M. Haider, "An Approach to Implement Frees Space Optical (FSO) Technology for Smart Village Energy Autonomous Systems," Far East Journal of Electronics and Communications, vol. 18, 032018

[146] A.S. Hamza, "Optical wireless communications for airport surface operations: Opportunities and challenges," 2016 Integrated Communications Navigation and Surveillance (ICNS), pp. 2B3-1-2B3-7, April 2016.

[147] L.I. Albraheem et al., "Toward Designing a Li-Fi-Based Hierarchical IoT Architecture," IEEE Access, vol. 6, pp. 40 811-40 825, 2018.

[148] M. Ruo Roch and M. Martina, "Integrated Light Sensing and Communication for LED Lighting," Designs, vol. 2, no. 4, 2018.

[149] P. Sul'aj et al., "An Example of Li-Fi Technology Implementation for Home Automation," 2018 World Symposium on Digital Intelligence for Systems and Machines (DISA), pp. 183-187, Aug 2018.

[150] V. Matus et al., "Implementation of a Low-Cost Vehicular VLC System and CAN Bus Interface," 2018 11th International Symposium on Communication Systems, Networks Digital Signal Processing (CSNDSP), pp. 1-5, July 2018.

[151] W. Boubakri et al., "An Optical Wireless Communication Based 5G Architecture to Enable Smart City Applications," 2018 20th International Conference on Transparent Optical Networks (ICTON), pp. 1-6, July 2018

[152] Z. Ong and W. Chung, "Long Range VLC Temperature Monitoring System Using CMOS of Mobile Device Camera," IEEE Sensors Journal, vol. 16, no. 6, pp. 1508-1509, March 2016.

[153] T. Zhou et al., "Temperature Monitoring System Based on Hadoop and VLC," Procedia Computer Science, vol. 131, pp. 1346-1354, 2018.

[154] M.D. Thieu et al., "Optical-RoI-Signaling for Vehicular Communications," IEEE Access, vol. 7, pp. 69 873-69 891, 2019.

[155] M.K. Hasan et al., "Simultaneous Traffic Sign Recognition and RealTime Communication using Dual Camera in ITS," 2019 International Conference on Artificial Intelligence in Information and Communication (ICAIIC), pp. 517-520, Feb 2019.

[156] T. Tripp et al., "Low-Cost UFSOOK-Based Optical Camera Communication Link for IoT Applications," IEEE 6th World Forum on Internet of Things, 2020.

[157] C. SaiSankeerth Reddy et al., "Appliance Automation Using LiFi with MQTT in Hybrid Star amp; Tree Network Setup," 2018 International Conference on Research in Intelligent and Computing in Engineering (RICE), pp. 1-6, Aug 2018.

[158] H. Lee et al., "Optical Wireless Sensor Networks Based on VLC with PLC-Ethernet Interface," International Journal of Electrical, Computer, Energetic, Electronic and Communication Engineering, vol. 5, no. 9, pp. 1204-1207, 2011.

[159] L. Li et al., "Epsilon: A visible light based positioning system," in 11th \{USENIX\} Symposium on Networked Systems Design and Implementation (\{NSDI\} 14), 2014, pp. 331-343.

[160] K. Warmerdam et al., "Connectivity in IoT indoor lighting systems with visible light communications," 2015 IEEE Online Conference on Green Communications (OnlineGreenComm), pp. 47-52, Nov 2015.

[161] Y. Shen et al., "Poster: A VLC Solution for Smart Parking," Proceedings of the 23rd Annual International Conference on Mobile Computing and Networking, pp. 579-581, 2017.

[162] V. Mariappan et al., "IoL Field Gateway : An Integrated IoT Agent using Networked Smart LED Lighting Controller," The Journal of The Korean Institute of Communication Sciences, vol. 34, pp. 12-19, 2017. 
[163] C.M. Kim and S.J. Koh, "Device Management and Data Transport in IoT Networks Based on Visible Light Communication," Sensors, vol. 18 , no. $8,2018$.

[164] M. Novak et al., "On human to database interface based on visible light communication," 2018 Global LIFI Congress (GLC), pp. 1-4, Feb 2018.

[165] Y. Almadani et al., "Application of Visible Light Communication in an Industrial Environment," 2018 11th International Symposium on Communication Systems, Networks Digital Signal Processing (CSNDSP), pp. 1-6, July 2018.

[166] P.W. Berenguer et al., "Real-Time Optical Wireless Communication: Field-Trial in an Industrial Production Environment," 2018 European Conference on Optical Communication (ECOC), pp. 1-3, Sep. 2018.

[167] P.W. Berenguer et al., "Real-Time Optical Wireless Mobile Communication With High Physical Layer Reliability," Journal of Lightwave Technology, vol. 37, no. 6, pp. 1638-1646, March 2019.

[168] H. Haas, "LiFi is a paradigm-shifting 5G technology," Reviews in Physics, vol. 3, pp. 26-31, 2018. [Online]. Available: http: //www.sciencedirect.com/science/article/pii/S2405428317300151

[169] X. Bao et al., "Li-Fi: Light fidelity-a survey," Wireless Networks, vol. 21, no. 6, pp. 1879-1889, Aug 2015. [Online]. Available: https://doi.org/10.1007/s11276-015-0889-0

[170] S. Pervez et al., "LiFi: The Future for Indoor Wireless Data Communication," International Journal of Scientific and Engineering Research, vol. 7, pp. 71-79, 102016

[171] S. Jung et al., "TDOA-based optical wireless indoor localization using LED ceiling lamps," IEEE Transactions on Consumer Electronics, vol. 57, no. 4, pp. 1592-1597, November 2011.

[172] T.H. Do and M. Yoo, "An in-Depth Survey of Visible Light Communication Based Positioning Systems," Sensors, vol. 16, no. 5, 2016. [Online]. Available: http://www.mdpi.com/1424-8220/16/5/678

[173] X. Guo et al., "Indoor Localization Using Visible Light Via Fusion of Multiple Classifiers," IEEE Photonics Journal, vol. 9, no. 6, pp. 1-16, Dec 2017.

[174] Y. Li et al., "A VLC smartphone camera based indoor positioning system," IEEE Photonics Technology Letters, vol. 30, no. 13, pp. 11711174, July 2018.

[175] Y. Zhuang et al., "A Survey of Positioning Systems Using Visible LED Lights," IEEE Communications Surveys Tutorials, vol. 20, no. 3, pp. 1963-1988, thirdquarter 2018.

[176] N. Rajagopal et al., "Hybrid Visible Light Communication for Cameras and Low-power Embedded Devices," Proceedings of the 1st ACM MobiCom Workshop on Visible Light Communication Systems, pp. 33-38, 2014. [Online]. Available: http://doi.acm.org/10.1145/ 2643164.2643173

[177] Y. Wang et al., "4.5-Gb/s RGB-LED based WDM visible light communication system employing CAP modulation and RLS based adaptive equalization," Optics Express, vol. 23, no. 10, pp. 1362613633, May 2015. [Online]. Available: http://www.opticsexpress.org/ abstract.cfm?URI=oe-23-10-13626
[178] L. Incipini et al., "Sensing Light with LEDs: Performance Evaluation for IoT Applications," Journal of Imaging, vol. 3, no. 4, 2017. [Online]. Available: http://www.mdpi.com/2313-433X/3/4/50

[179] J. Li et al., "Large-coverage underwater visible light communication system based on blue LED employing equal gain combining with integrated PIN array reception," Appl. Opt., vol. 58, no. 2, pp. 383-388, Jan 2019.

[180] J. Li et al., "Retro-VLC: Enabling Battery-free Duplex Visible Light Communication for Mobile and IoT Applications," Proceedings of the 16th International Workshop on Mobile Computing Systems and Applications, pp. 21-26, 2015.

[181] S. Ma et al., "Foglight: Visible Light-Enabled Indoor Localization System for Low-Power IoT Devices," IEEE Internet of Things Journal, vol. 5, no. 1, pp. 175-185, Feb 2018.

[182] S. Shao et al., "Passiveretro: Enabling completely passive visible light localization for iot applications," IEEE INFOCOM 2019 - IEEE Conference on Computer Communications, pp. 1540-1548, April 2019.

[183] A.B.M.M. Rahman et al., "Recent advances in indoor localization via visible lights: A survey," Sensors, vol. 20, no. 5, 2020. [Online]. Available: https://www.mdpi.com/1424-8220/20/5/1382

[184] I. Demirkol et al., "Powering the Internet of Things through Light Communication," IEEE Communications Magazine, vol. 57, no. 6, pp. 107-113, June 2019

[185] F. Delgado-Rajo et al., "Hybrid rf/vlc network architecture for the internet of things," Sensors, vol. 20, no. 2, 2020. [Online]. Available: https://www.mdpi.com/1424-8220/20/2/478

[186] M.Z. Chowdhury et al., "Optical wireless hybrid networks: Trends, opportunities, challenges, and research directions," IEEE Communications Surveys Tutorials, vol. 22, no. 2, pp. 930-966, 2020.

[187] X. Guo et al., "PSync: Visible light-based time synchronization for Internet of Things (IoT)," IEEE INFOCOM 2016 - The 35th Annual IEEE International Conference on Computer Communications, pp. 19, April 2016.

[188] C. Kim et al., "IDMP-VLC: IoT device management protocol in visible light communication networks," 2017 19th International Conference on Advanced Communication Technology (ICACT), pp. 578-583, Feb 2017.

[189] D. Giustiniano et al., "Connecting Battery-free IoT Tags Using LED Bulbs," Proceedings of the 17th ACM Workshop on Hot Topics in Networks, pp. 99-105, 2018.

[190] M.K. Hasan et al., "Performance analysis and improvement of optical camera communication," Applied Sciences, vol. 8, no. 12, 2018. [Online]. Available: https://www.mdpi.com/2076-3417/8/12/2527

[191] Z. Tian et al., "The darkLight Rises: Visible Light Communication in the Dark," Proceedings of the 22Nd Annual International Conference on Mobile Computing and Networking, pp. 2-15, 2016. [Online]. Available: http://doi.acm.org/10.1145/2973750.2973772

[192] Z. Tian et al., "Lighting Up the Internet of Things with DarkVLC," Proceedings of the 17th International Workshop on Mobile Computing Systems and Applications, pp. 33-38, 2016. 